\title{
A loosely-coupled scheme for the interaction between a fluid, elastic structure and poroelastic material
}

\author{
M. Bukača \\ ${ }^{a}$ Department of Applied and Computational Mathematics and Statistics, University of Notre Dame, 158 \\ Hurley Hall, Notre Dame, IN 46556, USA
}

\begin{abstract}
We model the interaction between an incompressible, viscous fluid, thin elastic structure and a poroelastic material. The poroelastic material is modeled using the Biot's equations of dynamic poroelasticity. The fluid, elastic structure and the poroelastic material are fully coupled, giving rise to a nonlinear, moving boundary problem with novel energy estimates. We present a modular, loosely coupled scheme where the original problem is split into the fluid sub-problem, elastic structure sub-problem and poroelasticity sub-problem. An energy estimate associated with the stability of the scheme is derived in the case where one of the coupling parameters, $\beta$, is equal to zero. We present numerical tests where we investigate the effects of the material properties of the poroelastic medium on the fluid flow. Our findings indicate that the flow patterns highly depend on the storativity of the poroelastic material and can not be captured by considering fluid-structure interaction only.
\end{abstract}

Keywords: fluid-structure interaction, poroelasticity, fluid-porous media interaction, loosely-coupled scheme, blood clots

\section{Introduction}

Poroelastic materials, due to their dissipative properties, are extensively used in the automotive industry, aeronautics, and building acoustics for the purpose of noise control. They are also ubiquitous in nature. Examples include bones, soil, blood clots and biological tissues. In practical applications poroelastic materials are involved in multilayered structures comprising elastic media. These materials are exposed to a wide spectrum of dynamic loads. In the aerospace industry, dam-reservoir systems, off-shore structures and in many biological systems, the dynamic loading comes from the surrounding fluid such as air, water or blood flow. Understanding the interaction between a fluid, elastic structure and a poroelastic material is important for the understanding of the normal function and prevention of damage and catastrophic events in many applications. One of the applications of such systems in hemodynamics is the interaction of a blood clot with blood flow dynamics and vessel wall mechanics. Understanding their interaction plays an important role in making decisions about medical treatment, since it helps identify flow conditions which may lead to medical complications. In this work, we focus on this application and show that accurate flow patterns can not

Email address: mbukac@nd.edu (M. Bukač) 
be captured without including the dynamics of the blood clot in fluid-structure interaction models.

Blood clots contain different types of blood [1], cells [32] and extracellular matrix constituents. Near the vessel wall, they show a considerable amount of collagen [44], forming a core with a low permeability. The layers around the core contain mainly fibers, overlaid by a micro-structure of pores and cavities filled by a fluid $[1,36]$ whose dimensions vary strongly. Blood clots have been shown to be permeable to fluids, which potentially allows the passage of macromolecules to the vessel wall [1]. Hence, the blood clot dynamics on a macro scale have been previously described using porous media models [26, 65], and poroelasticity models [57]. However, none of these approaches considered the full interaction between blood, blood clot and the vessel wall. The work presented here is the first step in that direction.

In the existing literature, problems involving the interaction between a fluid, elastic structure and poroelastic material are predominantly studied in terms of the interaction of poroelastic media with an elastic structure and an air-filled acoustic region $[3,59,4,24,67,48,46$, 43]. In contrast to the existing work related to acoustic wave propagation, we are interested in the interaction between an incompressible, viscous fluid, an elastic structure and a poroelastic material with applications to hemodynamics. The novel, two-way coupling gives rise to a non-linear, moving boundary problem with energy estimates not considered before. Since the coupled problem features interaction between different physical phenomena, defined on separate domains, we develop and analyze a loosely-coupled numerical algorithm that captures this interaction. We split the fully coupled problem into a fluid sub-problem, elastic structure sub-problem and poroelasticity sub-problem. At every time step, each of these sub-problems is solved only once, without additional iterations between the sub-problems, leading to a loosely-coupled scheme. To derive the numerical scheme, we extend and combine splitting strategies for fluid-structure interaction (FSI) problems and fluid-poroelastic material interaction (FPI) problems.

The development of partitioned numerical methods for FSI problems has been extensively studied in the literature $[11,6,52,28,27,55,39,8,58,47]$. However, one of the main concerns of partitioned schemes is stability. It has been shown in [20] that "classical" partitioned strategies suffer from numerical instabilities known as the added mass effect when applied to problems in hemodynamics. To resolve this issue, several alternative methods have been proposed. Methods proposed in $[31,55]$ use a membrane model for the structure that is then embedded into the fluid problem where it appears as a generalized Robin boundary condition. We further mention the numerical schemes proposed in $[6,5]$ where the fluid and structure were split in the classical way, but the coupling conditions were combined in a novel way that improved the convergence rate. A different approach based on Nitsche's penalty method [39] was used in $[14,15]$.

Recently, a loosely coupled scheme, called the "kinematically coupled $\beta$-scheme," was introduced in [11]. This scheme successfully deals with stability problems associated with the added mass effect in a novel way. The scheme originates from the "classical" kinematically coupled scheme introduced in [38], where a parameter $\beta$ was introduced in [11] to increase the accuracy of the scheme. The kinematically coupled scheme introduced in [38] corresponds to the case when $\beta=0$. Taking $\beta>0$, and in particular $\beta=1$, is shown to increase the accuracy without affecting the stability [11]. Stability and accuracy results on a nonlinear benchmark FSI problem in [11] show that the accuracy of the kinematically coupled $\beta$ - 
scheme with $\beta=1$ is comparable to the accuracy of a monolithic scheme by Badia, Quaini, and Quarteroni [7]. Stability was further studied analytically in [16], where unconditional stability for any $\beta \in[0,1]$ was shown. The stability is achieved by combining the structure inertia with the fluid sub-problem as a Robin boundary condition in a novel way, which mimics the energy balance of the continuous problem.

A class of incremental displacement-correction schemes for the explicit coupling of FSI problems has been introduced in [29, 28]. The non-incremental variant of the algorithm presented there corresponds to the kinematically coupled scheme, while the incremental scheme consists of treating the structure displacement explicitly in the fluid sub-problem and then correcting it in the structure sub-problem. The authors in [28] presented stability and convergence analysis for FSI problems with thin structures, for the whole class of incremental displacement-correction schemes, showing that the incremental scheme features optimal convergence, while sub-optimal convergence is expected for the original non-incremental variant. In [29] the authors proposed a generalization of the explicit coupling schemes reported in $[28,38]$ to the case of FSI with thick structures.

The kinematically coupled $\beta$ scheme was also used in a modified version by Lukacova et al. in $[47,42]$ to study FSI involving non-Newtonian fluids, and by Bukač et al. in [12] to model the interaction between a fluid and a composite structure. These recent results indicate that the kinematically-coupled $\beta$ scheme and its modifications provide an appealing way to solve FSI problems using a partitioned approach and therefore we employ it in this work.

In addition to the interaction between fluid and an elastic structure discussed above, the model we study also features the interaction between the fluid and a poroelastic material. A poroelastic material consists of an elastic skeleton and connecting pores filled with fluid. A common way to describe such system is using a Biot model. Due to the various geomechanical applications, where the structure dynamics is negligible, the quasi-static Biot model is well studied in the literature $[64,61,62,56,49]$. However, since we are interested in hemodynamics applications in which the structure dynamics are significant, we consider the dynamic Biot model $[21,66]$.

The coupling between a fluid and a poroelastic medium has been previously studied in $[34,8,45,60,53,63,37,35,13]$. The formulation, boundary conditions and well-posedness were studied in [60]. Numerical studies include the work in [8], where the FPI problem is solved using both a monolithic and a partitioned approach. The partitioned approach is based on the domain decomposition procedure. However, sub-iterations are needed between the two problems due to the instabilities associated with the added mass effect. Studies in [37, 35] considered coupling between a poroelastic material and a reduced fluid model described by the lubrication equation in order to study the flow in fractures. Particular applications of the FPI model were studied in [45], where such a system was used to describe the blood flow and low-density lipoprotein transport in a porohyperelastic arterial wall, and in [63] to model cerebrospinal fluid hydrodynamics.

In this work, in order to split the fluid problem from the dynamic poroelasticity problem, we extend and adapt the splitting strategy applied in [18] to the Stokes/Darcy interaction problem. Furthermore, similar to [8], we introduce combination parameters, which are then combined with the splitting used in [18], and applied to the interaction between the fluid and a poroelastic material. The splitting strategy from [18] allows a design of a loosely coupled 
scheme, while the introduction of combination parameters helps to improve the accuracy of values at the fluid-poroelastic material interface.

To split the fluid problem from the elastic structure problem, we use the kinematically coupled $\beta$-scheme. Finally, we design a splitting strategy in the spirit of the kinematically coupled $\beta$-scheme to split the thin elastic structure problem from the dynamic poroelasticity problem. In order to achieve stability, we split the thin structure equation so that the structure inertia is included in both the fluid sub-problem and the poroelasticity sub-problem as a Robin boundary condition. In this way, the scheme does not suffer from instabilities associated with the added mass effect.

The model equations and the fully coupled problem with novel energy estimates are presented in Section 2. The loosely coupled scheme and the stability analysis are given in Sections 3 and 4, respectively. Numerical results, presented in Section 5, focus on the verification of the stability analysis, as well as on testing the significance of the model. We also investigate the effects of the material properties of the poroelastic medium on the fluid flow. We show that the fully coupled fluid/structure/poroelastic material model features flow patters which can not be recovered by modeling fluid-structure interaction only. Moreover, the flow patterns heavily depend on the storativity properties of the poroelastic material.

\section{Mathematical model}

\subsection{Problem Definition}

Consider a bounded, deformable domain $\Omega(t)=\Omega^{f}(t) \cup \Omega^{p}(t)$ in $\mathbb{R}^{d}, d=2,3$, which consists of two regions, $\Omega^{f}(t)$ and $\Omega^{p}(t)$, see Figure 1 . We assume that the reference domain
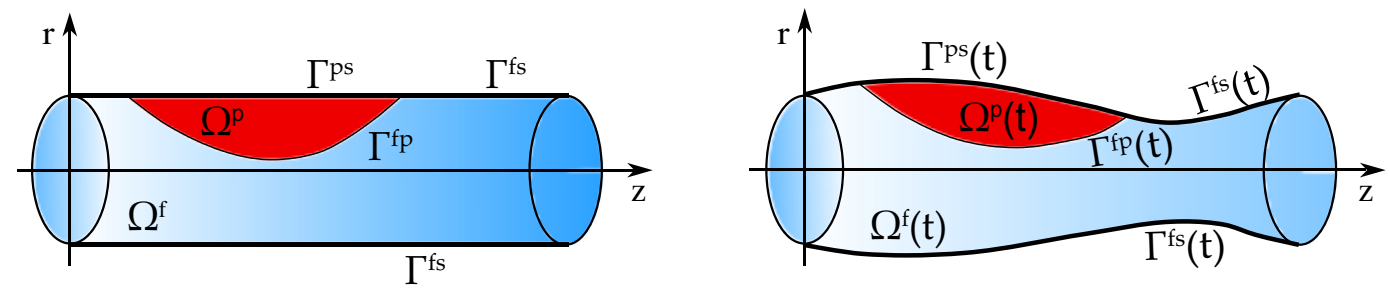

Figure 1: Left: Reference domains $\Omega=\Omega^{f} \cup \Omega^{p}$. Right: Deformed domains $\Omega(t)=\Omega^{f}(t) \cup \Omega^{p}(t)$.

$\Omega$ is a cylinder of radius $R$ and length $L$. The lateral boundary of the cylinder represents a thin, elastic wall, whose reference domain is denoted by $\Gamma^{s}=\Gamma^{f s} \cup \Gamma^{p s}$.

The reference domain $\Omega^{f}$ is filled with an incompressible, viscous fluid, and the reference domain $\Omega^{p}$ is occupied by a fully-saturated poroelastic matrix. The poroelastic matrix is immersed in the fluid, and in contact with the elastic structure. The interface between the fluid and the poroelastic matrix is denoted by $\Gamma^{f p}(t)$, the fluid-structure interface is denoted by $\Gamma^{f s}(t)$, while the interface between the poroelastic matrix and the structure is denoted by $\Gamma^{p s}(t)$. We are interested in simulating a pressure-driven flow through the deformable channel with a two-way coupling between the fluid, elastic structure and poroelastic material.

We model the flow in $\Omega^{f}(t)$ using the Navier-Stokes equations for a viscous, incompressible, Newtonian fluid, which in the Eulerian formulation reads as follows:

$$
\rho_{f}\left(\partial_{t} \boldsymbol{v}+\boldsymbol{v} \cdot \nabla \boldsymbol{v}\right)=\nabla \cdot \boldsymbol{\sigma}^{f} \quad \text { in } \Omega^{f}(t) \times(0, T),
$$




$$
\nabla \cdot \boldsymbol{v}=0 \quad \text { in } \Omega^{f}(t) \times(0, T),
$$

where $\boldsymbol{v}$ is the fluid velocity, $\boldsymbol{\sigma}^{f}=-p_{f} \boldsymbol{I}+2 \mu_{f} \boldsymbol{D}(\boldsymbol{v})$ is the fluid stress tensor, $p_{f}$ is the fluid pressure, $\rho_{f}$ is the fluid density, $\mu_{f}$ is the fluid viscosity and $\boldsymbol{D}(\boldsymbol{v})=\left(\nabla \boldsymbol{v}+(\nabla \boldsymbol{v})^{T}\right) / 2$ is the rate-of-strain tensor. Denote the inlet and outlet fluid boundaries by $\Gamma_{\text {in }}^{f}$ and $\Gamma_{\text {out }}^{f}$, respectively. In practice, at the inlet and outlet boundaries we prescribe the normal stress:

$$
\begin{array}{lr}
\boldsymbol{\sigma}^{f} \boldsymbol{n}_{\text {in }}^{f}=-p_{\text {in }}(t) \boldsymbol{n}_{\text {in }}^{f} & \text { on } \Gamma_{\text {in }}^{f} \times(0, T), \\
\boldsymbol{\sigma}^{f} \boldsymbol{n}_{\text {out }}^{f}=-p_{\text {out }}(t) \boldsymbol{n}_{\text {in }}^{f} & \text { on } \Gamma_{\text {out }}^{f} \times(0, T),
\end{array}
$$

where $\boldsymbol{n}_{\text {in }}^{f}$ and $\boldsymbol{n}_{\text {out }}^{f}$ are the outward normals to the inlet and outlet fluid boundaries, respectively. These boundary conditions are common in blood flow modeling [5, 50, 54]. For theoretical purposes we also consider the dynamic pressure boundary conditions with zero tangential velocity

$$
\begin{array}{ll}
p_{f}+\frac{\rho_{f}}{2}|\boldsymbol{v}|^{2}=p_{\text {in } / \text { out }}(t) & \text { on } \Gamma_{\text {in } / \text { out }}^{f} \times(0, T), \\
\boldsymbol{v} \times \boldsymbol{e}_{z}=0 & \text { on } \Gamma_{\text {in } / \text { out }}^{f} \times(0, T) .
\end{array}
$$

The cylinder lateral boundary $\Gamma^{s}(t)$ represents a deformable, elastic wall. We assume that the wall behaves as a linear, thin solid (e.g. string, membrane, shell), whose Lagrangian formulation, in the first order form, can be written as

$$
\begin{aligned}
\rho_{m} h \partial_{t} \boldsymbol{\xi}+\mathcal{L} \boldsymbol{\eta}=\boldsymbol{f} & \text { on } \Gamma^{s} \times(0, T), \\
\boldsymbol{\xi}=\partial_{t} \boldsymbol{\eta} & \text { on } \Gamma^{s} \times(0, T) .
\end{aligned}
$$

where $\mathcal{L}$ is a linear differential operator derived from the elastic energy of the thin structure. Specific choices of $\mathcal{L}$ will be made in Section 5. The structure displacement is denoted by $\boldsymbol{\eta}$, and the structure velocity by $\boldsymbol{\xi}$. The structure density is denoted by $\rho_{m}$, the structure thickness by $h$, and the vector of the surface density of the force applied to the shell by $\boldsymbol{f}$.

The fluid domain and the elastic structure are in contact with a deformable porous matrix consisting of an elastic skeleton and the connecting pores filled with fluid. Its dynamics is described by the Biot's equations of dynamic poroelasticity $[21,4,24,48,8]$, which in the Lagrangian formulation read as follows

$$
\begin{array}{ll}
\rho_{b} \partial_{t} \boldsymbol{V}+\rho_{q} \partial_{t} \boldsymbol{q}=\nabla \cdot \boldsymbol{\sigma}^{p} & \text { in } \Omega^{p} \times(0, T), \\
\boldsymbol{\kappa}^{-1} \boldsymbol{q}=-\nabla p_{p}-\rho_{q} \partial_{t} \boldsymbol{V}-m \partial_{t} \boldsymbol{q} & \text { in } \Omega^{p} \times(0, T), \\
\frac{1}{M} \partial_{t} p_{p}+\alpha \nabla \cdot \boldsymbol{V}+\nabla \cdot \boldsymbol{q}=0 & \text { in } \Omega^{p} \times(0, T),
\end{array}
$$

where $\boldsymbol{U}$ and $\boldsymbol{V}=\partial_{t} \boldsymbol{U}$ are the displacement and the velocity of the solid skeleton, respectively. The average velocity of fluid relative to the velocity of the solid is denoted by $\boldsymbol{q}=\phi\left(\boldsymbol{V}_{f}-\boldsymbol{V}\right)$, where $\boldsymbol{V}_{f}$ denotes the velocity of the fluid in the pores and $\phi$ is the porosity. The fluid pore pressure is denoted by $p_{p}$. The stress tensor of the poroelastic medium is given by $\boldsymbol{\sigma}^{p}=\boldsymbol{\sigma}^{E}(\boldsymbol{U})-\alpha p_{p} \boldsymbol{I}$, where $\boldsymbol{\sigma}^{E}(\boldsymbol{U})$ denotes the elasticity stress tensor. For the Saint Vernant-Kirchoff elastic material, $\boldsymbol{\sigma}^{E}(\boldsymbol{U})=2 \mu_{s} \boldsymbol{D}(\boldsymbol{U})+\lambda_{s}(\nabla \cdot \boldsymbol{U}) \boldsymbol{I}$, where $\lambda_{s}$ and $\mu_{s}$ denote 
the Lamé coefficients for the elastic phase. Given the density of the solid material $\rho_{s}$ and the fluid density $\rho_{q}$, the density of the solid-fluid mixture is defined by $\rho_{b}=(1-\phi) \rho_{s}+\phi \rho_{q}$. The uniformly positive definite permeability tensor is denoted by $\boldsymbol{\kappa}$, and $m=\frac{1}{\phi} \rho_{q}$ [68]. Finally, $\alpha$ and $M$ are the Biot parameters accounting for the compressibility in the two-phase material. The Biot modulus $M$ is the inverse of a storage coefficient, defined as the increase of the amount of fluid as a result of a unit increase of pore pressure under constant volumetric strain. System (2.9)-(2.11) consists of the equlibrium equation for the balance of total forces (2.9), the generalized Darcy's law (2.10) and the continuity equation (2.11).

Initially, the fluid, elastic structure and the poroelastic material are assumed to be at rest with zero displacement form the reference configuration:

$$
\boldsymbol{v}=0, \boldsymbol{\eta}=0, \boldsymbol{\xi}=0, \boldsymbol{U}=0, \boldsymbol{V}=0, \boldsymbol{q}=0 \text { and } p_{p}=0 .
$$

\subsubsection{The coupling conditions}

Denote by $\boldsymbol{n}^{f}$ the outward normal to the fluid domain and by $\left\{\boldsymbol{\tau}_{1}^{f}, \boldsymbol{\tau}_{2}^{f}\right\}$ the corresponding tangential unit vectors. Since the fluid equations are defined in the physical domain, and poroelastic and elastic structure equations are given with respect to the reference domain, we denote the Jacobian of the transformation from the Eulerian to the Lagrangian coordinate system by $J$. In order to define the fully coupled problem, we prescribe the coupling conditions between the fluid and elastic structure on $\Gamma^{f s}$, between the fluid and poroelastic material on $\Gamma^{f p}$, and between the poroelastic material and elastic structure on $\Gamma^{p s}$.

Fluid-structure interaction: To capture the interaction between the fluid and elastic structure, we prescribe the kinematic and dynamic coupling conditions [51, 55, 7]:

The continuity of velocities:

The conservation of momentum:

$$
\begin{array}{rlrl}
\left.\boldsymbol{v}\right|_{\Gamma^{f s}(t)} & =\boldsymbol{\xi} & & \text { on } \Gamma^{f s} \times(0, T), \\
-\left.J \boldsymbol{\sigma}^{f} \boldsymbol{n}^{f}\right|_{\Gamma^{f s}(t)}=\boldsymbol{f} & \text { on } \Gamma^{f s} \times(0, T) .
\end{array}
$$

Fluid-poroelastic structure interaction: To couple the fluid and poroelastic structure, we prescribe the continuity of normal flux, the Beavers-Joseph-Saffman coupling condition where the tangential component of the normal fluid stress is proportional to the jump in the tangential velocity over the interface $\Gamma^{f p}$ with the slip rate $\gamma^{f p}$, the conservation of momentum for the fluid phase, and the balance of contact forces $[60,53,8]$ :

$$
\begin{array}{lr}
\text { Mass conservation: } & \left.\boldsymbol{v} \cdot \boldsymbol{n}^{f}\right|_{\Gamma^{f p}(t)}=(\boldsymbol{V}+\boldsymbol{q}) \cdot \boldsymbol{n}^{f} \text { on } \Gamma^{f p} \times(0, T), \\
\text { Slip condition: } & \left.J \boldsymbol{\tau}_{i}^{f} \cdot \boldsymbol{\sigma}^{f} \boldsymbol{n}^{f}\right|_{\Gamma^{f p}(t)}=-\gamma^{f p}\left(\left.\boldsymbol{v}\right|_{\Gamma^{f p}(t)}-\boldsymbol{V}\right) \cdot \boldsymbol{\tau}_{i}^{f} \\
i & =1,2, \text { on } \Gamma^{f p} \times(0, T), \\
& \begin{array}{r}
\text { Momentum conservation: } \\
\text { Balance of contact forces: }
\end{array} \\
\left.J \boldsymbol{n}^{f} \cdot \boldsymbol{\sigma}^{f} \boldsymbol{n}^{f}\right|_{\Gamma^{f p}(t)}=-p_{p} \text { on } \Gamma^{f p} \times(0, T), \\
\left.J \boldsymbol{\sigma}^{f} \boldsymbol{n}^{f}\right|_{\Gamma^{f p}(t)}=\boldsymbol{\sigma}^{p} \boldsymbol{n}^{f} \text { on } \Gamma^{f p} \times(0, T) .
\end{array}
$$

Elastic-poroelastic structure interaction: Denote by $\boldsymbol{n}^{p}$ the outward normal vector to $\Omega^{p}$ and by $\left\{\boldsymbol{\tau}_{1}^{p}, \boldsymbol{\tau}_{2}^{p}\right\}$ the corresponding tangential unit vectors. In order to define the coupling between the elastic and poroelastic structure, we follow the approach in $[2,24,48]$ and assume that there is no relative mass flux across the boundary $\Gamma^{p s}$, the continuity of displacement, and the conservation of momentum:

Zero relative mass flux:

$$
\boldsymbol{q} \cdot \boldsymbol{n}^{p}=0 \quad \text { on } \Gamma^{p s} \times(0, T)
$$




$$
\begin{array}{lll}
\text { The continuity of displacement: } & \boldsymbol{U}=\boldsymbol{\eta} & \text { on } \Gamma^{p s} \times(0, T), \\
\text { Balance of contact forces: } & \boldsymbol{f}=-\boldsymbol{\sigma}^{p} \boldsymbol{n}^{p} & \text { on } \Gamma^{p s} \times(0, T) .
\end{array}
$$

Remark 1. Note that the Dirichlet conditions (2.13), (2.15), (2.19) and (2.20) are compatible at the intersection of $\Gamma^{f s}, \Gamma^{f p}$ and $\Gamma^{p s}$. Moreover, if the primal mixed formulation is used for the Biot system, it is enough to strongly impose only (2.13) and (2.20) in the monolithic problem.

\subsection{Weak formulation and the energy estimates of the monolithic problem}

In this section we derive the weak formulation and energy estimates of the coupled problem. Since the fluid equations are given in the Eulerian coordinates while the elastic and poroelastic structure equations are given in the Lagrangian coordinates, we use the Arbitrary Lagrangian-Eulerian (ALE) method in order to deal with the motion of the fluid domain.

\subsection{The ALE mapping}

The ALE approach $[41,25,54]$ is based on introducing a family of (arbitrary, invertible, smooth) mappings $\mathcal{A}_{t}$ defined on the reference domain $\Omega^{f}$ such that for each $t \in\left(t_{0}, T\right), \mathcal{A}_{t}$ maps the reference domain $\Omega^{f}$ into the physical domain $\Omega^{f}(t)$. In the context of the finite element approximation of moving-boundary problems with "small" displacement, the ALE method deals efficiently with the deformation of the mesh, and with the issues related to the approximation of the time-derivatives. We define $\mathcal{A}_{t}$ to be a harmonic extension of the mapping $\tilde{g}$ that maps the boundary of $\Omega^{f}$ to the boundary of $\Omega^{f}(t)$ for a given time $t$ :

$$
\begin{array}{rll}
\Delta \mathcal{A}_{t} & =0 & \text { in } \Omega^{\mathrm{f}}, \\
\mathcal{A}_{t} & =\tilde{g} & \text { on } \Gamma^{\mathrm{fp}} \cup \Gamma^{\mathrm{fs}}, \\
\mathcal{A}_{t} & =0 & \text { on } \partial \Omega^{\mathrm{f}} \backslash\left(\Gamma^{\mathrm{fp}} \cup \Gamma^{\mathrm{fs}}\right) .
\end{array}
$$

Denoting the domain velocity by $\boldsymbol{w}=\partial_{t} \mathcal{A}_{t}$, the Navier-Stokes equations in the ALE framework read as follows

$$
\begin{array}{ll}
\rho_{f}\left(\left.\frac{\partial \boldsymbol{v}}{\partial t}\right|_{\hat{\boldsymbol{x}}}+(\boldsymbol{v}-\boldsymbol{w}) \cdot \nabla \boldsymbol{v}\right)=\nabla \cdot \boldsymbol{\sigma}^{f} & \text { in } \Omega^{f}(t) \times(0, T), \\
\nabla \cdot \boldsymbol{v}=0 & \text { in } \Omega^{f}(t) \times(0, T) .
\end{array}
$$

\subsection{Weak formulation of the monolithic problem}

We start by introducing the following bilinear forms:

$$
\begin{aligned}
a_{f}\left(\boldsymbol{v}, \boldsymbol{\varphi}^{f}\right) & =2 \mu_{f} \int_{\Omega^{f}(t)} \boldsymbol{D}(\boldsymbol{v}): \boldsymbol{D}\left(\boldsymbol{\varphi}^{f}\right) d \boldsymbol{x}, \\
b_{f}\left(p_{f}, \boldsymbol{\varphi}^{f}\right) & =\int_{\Omega^{f}(t)} p_{f} \nabla \cdot \boldsymbol{\varphi}^{f} d \boldsymbol{x}, \\
d_{f}\left(\boldsymbol{v}, \boldsymbol{v}, \boldsymbol{\varphi}^{f}\right) & =\rho_{f} \int_{\Omega^{f}(t)}(\boldsymbol{v} \cdot \nabla) \boldsymbol{v} \cdot \boldsymbol{\varphi}^{f} d \boldsymbol{x}, \\
a_{m}(\boldsymbol{\eta}, \boldsymbol{\zeta}) & =\int_{\Gamma^{s}} \mathcal{L} \boldsymbol{\eta} \cdot \boldsymbol{\zeta} d x,
\end{aligned}
$$




$$
\begin{aligned}
a_{e}\left(\boldsymbol{U}, \varphi^{p}\right) & =2 \mu \int_{\Omega^{p}} \boldsymbol{D}(\boldsymbol{U}): \boldsymbol{D}\left(\boldsymbol{\varphi}^{p}\right) d \boldsymbol{x}+\lambda \int_{\Omega^{p}}(\nabla \cdot \boldsymbol{U})\left(\nabla \cdot \boldsymbol{\varphi}^{p}\right) d \boldsymbol{x}, \\
a_{q}\left(\boldsymbol{q}, \boldsymbol{\varphi}^{q}\right) & =\int_{\Omega^{p}} \boldsymbol{\kappa}^{-1} \boldsymbol{q} \cdot \boldsymbol{\varphi}^{q} d \boldsymbol{x}, \\
b_{q p}\left(p_{p}, \boldsymbol{\varphi}^{q}\right) & =\int_{\Omega^{p}} \nabla p_{p} \cdot \boldsymbol{\varphi}^{q} d \boldsymbol{x}, \\
b_{e p}\left(p_{p}, \boldsymbol{\varphi}^{p}\right) & =\alpha \int_{\Omega^{p}} p_{p} \nabla \cdot \boldsymbol{\varphi}^{p} d \boldsymbol{x}, \\
c_{p}\left(p_{p}, \boldsymbol{\varphi}^{p}\right) & =\int_{\Gamma^{f p}} p_{p} \boldsymbol{\varphi}^{p} \cdot \boldsymbol{n}^{f} d x, \\
c_{s}\left(\boldsymbol{V}, \boldsymbol{\varphi}^{p}\right) & =\int_{\Gamma^{f p}} \gamma^{f p} P_{\tau}^{f}(\boldsymbol{V}) P_{\tau}^{f}\left(\boldsymbol{\varphi}^{p}\right) d x,
\end{aligned}
$$

where $P_{\tau}^{f}(\cdot)$ is a projection onto the local tangential plane defined as

$$
P_{\tau}^{f}(\boldsymbol{V})=\boldsymbol{V}-\left(\boldsymbol{V} \cdot \boldsymbol{n}^{f}\right) \boldsymbol{n}^{f} .
$$

To find a weak form of the Navier-Stokes equations, introduce the following test function spaces:

$$
\begin{aligned}
& V^{f}(t)=\left\{\boldsymbol{\varphi}: \Omega^{f}(t) \rightarrow \mathbb{R}^{3} \mid \boldsymbol{\varphi}=\hat{\boldsymbol{\varphi}} \circ\left(\mathcal{A}_{t}\right)^{-1}, \hat{\varphi} \in\left(H^{1}\left(\Omega^{f}\right)\right)^{d}\right\}, \\
& Q^{f}(t)=\left\{\psi: \Omega^{f}(t) \rightarrow \mathbb{R} \mid \psi=\hat{\psi} \circ\left(\mathcal{A}_{t}\right)^{-1}, \hat{\psi} \in L^{2}\left(\Omega^{f}\right)\right\},
\end{aligned}
$$

for all $t \in[0, T)$. The variational formulation of the Navier-Stokes equations now reads: given $t \in(0, T)$ find $\left(\boldsymbol{v}, p_{f}\right) \in V^{f}(t) \times Q^{f}(t)$ such that for all $\left(\varphi^{f}, \psi^{f}\right) \in V^{f}(t) \times Q^{f}(t)$

$\rho_{f} \int_{\Omega^{f}(t)} \partial_{t} \boldsymbol{v} \cdot \varphi^{f} d \boldsymbol{x}+d_{f}\left(\boldsymbol{v}, \boldsymbol{v}, \varphi^{f}\right)+a_{f}\left(\boldsymbol{v}, \varphi^{f}\right)-b_{f}\left(p_{f}, \varphi^{f}\right)+b_{f}\left(\psi^{f}, \boldsymbol{v}\right)=\int_{\partial \Omega^{f}} \boldsymbol{\sigma}^{f} \boldsymbol{n}^{f} \cdot \varphi^{f} d x$.

In order to write the weak form of the thin structure model, let $V^{m}=\left(H_{0}^{1}\left(\Gamma^{s}\right)\right)^{d}$. Then the weak formulation reads as follows: given $t \in(0, T)$ find $\boldsymbol{\xi}=\partial_{t} \boldsymbol{\eta} \in V^{m}$ such that for all $\zeta \in V^{m}$

$$
\rho_{m} h \int_{\Gamma^{s}} \partial_{t} \boldsymbol{\xi} \cdot \boldsymbol{\zeta} d x+a_{m}(\boldsymbol{\eta}, \boldsymbol{\zeta})=\int_{\Gamma^{s}} \boldsymbol{f} \cdot \boldsymbol{\zeta} d x .
$$

Finally, we introduce $V^{p}=\left(H^{1}\left(\Omega^{p}\right)\right)^{d}, V^{q}=\left(L^{2}\left(\Omega^{p}\right)\right)^{d}$ and $Q^{p}=H^{1}\left(\Omega^{p}\right)$. The weak form of the dynamic Biot system (2.9)-(2.11) reads as follows: given $t \in(0, T)$ find $(\boldsymbol{V}=$ $\left.\partial_{t} \boldsymbol{U}, \boldsymbol{q}, p_{p}\right) \in V^{p} \times V^{q} \times Q^{p}$ such that for all $\left(\varphi^{p}, \varphi^{q}, \psi^{p}\right) \in V^{p} \times V^{q} \times Q^{p}$

$$
\begin{gathered}
\rho_{b} \int_{\Omega^{p}} \partial_{t} \boldsymbol{V} \cdot \boldsymbol{\varphi}^{p} d \boldsymbol{x}+\rho_{q} \int_{\Omega^{p}} \partial_{t} \boldsymbol{q} \cdot \boldsymbol{\varphi}^{p} d \boldsymbol{x}+a_{e}\left(\boldsymbol{U}, \boldsymbol{\varphi}^{p}\right)-b_{e p}\left(p_{p}, \boldsymbol{\varphi}^{p}\right)+a_{q}\left(\boldsymbol{q}, \boldsymbol{\varphi}^{q}\right)+b_{q p}\left(p_{p}, \boldsymbol{\varphi}^{q}\right) \\
+\rho_{q} \int_{\Omega^{p}} \partial_{t} \boldsymbol{V} \cdot \boldsymbol{\varphi}^{q} d \boldsymbol{x}+m \int_{\Omega^{p}} \partial_{t} \boldsymbol{q} \cdot \boldsymbol{\varphi}^{q} d \boldsymbol{x}+\int_{\Omega^{p}} \frac{1}{M} \partial_{t} p_{p} \psi^{p} d \boldsymbol{x}+b_{e p}\left(\psi^{p}, \boldsymbol{V}\right)-b_{q p}\left(\psi^{p}, \boldsymbol{q}\right) \\
=\int_{\partial \Omega^{p}} \boldsymbol{\sigma}^{p} \boldsymbol{n}^{p} \cdot \boldsymbol{\varphi}^{p} d x-\int_{\partial \Omega^{p}} \boldsymbol{q} \cdot \boldsymbol{n}^{p} \psi^{p} d x
\end{gathered}
$$


To write a weak formulation of the coupled fluid/structure/poroelastic material system, we define a space of admissible solutions

$$
W(t)=\left\{\left(\varphi^{f}, \boldsymbol{\zeta}, \varphi^{p}\right) \in V^{f}(t) \times V^{m} \times V^{p}\left|\varphi^{f}\right|_{\Gamma^{f s}(t)}=\boldsymbol{\zeta} \text { on } \Gamma^{f s}, \varphi^{p}=\boldsymbol{\zeta} \text { on } \Gamma^{p s}\right\},
$$

and add together equations (2.34), (2.35) and (2.36). We denote by $\mathcal{I}$ the sum of boundary integrals that appear on the right hand side

$$
\begin{gathered}
\mathcal{I}=\int_{\Gamma^{f s}(t)} \boldsymbol{\sigma}^{f} \boldsymbol{n}^{f} \cdot \boldsymbol{\varphi}^{f} d x+\int_{\Gamma^{f p}(t)} \boldsymbol{\sigma}^{f} \boldsymbol{n}^{f} \cdot \boldsymbol{\varphi}^{f} d x+\int_{\Gamma_{\text {in } / \text { out }}^{f}} \boldsymbol{\sigma}^{f} \boldsymbol{n}^{f} \cdot \boldsymbol{\varphi}^{f} d x+\int_{\Gamma^{f s}} \boldsymbol{f} \cdot \boldsymbol{\zeta} d x \\
+\int_{\Gamma^{p s}} \boldsymbol{f} \cdot \boldsymbol{\zeta} d x+\int_{\Gamma^{f p}} \boldsymbol{\sigma}^{p} \boldsymbol{n}^{p} \cdot \boldsymbol{\varphi}^{p} d x+\int_{\Gamma^{p s}} \boldsymbol{\sigma}^{p} \boldsymbol{n}^{p} \cdot \boldsymbol{\varphi}^{p} d x-\int_{\Gamma^{f p}} \boldsymbol{q} \cdot \boldsymbol{n}^{p} \psi^{p} d x-\int_{\Gamma^{p s}} \boldsymbol{q} \cdot \boldsymbol{n}^{p} \psi^{p} d x .
\end{gathered}
$$

Due to the zero relative mass flux condition (2.19), the last integral above is equal to zero. Using the change of variables, we recast the integrals over $\Gamma^{f s}(t)$ and $\Gamma^{f p}(t)$ into integrals over $\Gamma^{f s}$ and $\Gamma^{f p}$. Employing the kinematic conditions in (2.37) and condition (2.15), we have

$$
\begin{gathered}
\mathcal{I}=\int_{\Gamma^{f s}}\left(\left.J \boldsymbol{\sigma}^{f} \boldsymbol{n}^{f}\right|_{\Gamma^{f s}(t)}+\boldsymbol{f}\right) \cdot \boldsymbol{\zeta} d x+\int_{\Gamma^{p s}}\left(\boldsymbol{\sigma}^{p} \boldsymbol{n}^{p}+\boldsymbol{f}\right) \cdot \boldsymbol{\zeta} d x+\left.\left.\int_{\Gamma^{f p}} J \boldsymbol{\sigma}^{f} \boldsymbol{n}^{f}\right|_{\Gamma^{f p}(t)} \cdot \boldsymbol{\varphi}^{f}\right|_{\Gamma^{f p}(t)} d x \\
+\int_{\Gamma_{\text {in } / \text { out }}^{f}} \boldsymbol{\sigma}^{f} \boldsymbol{n}^{f} \cdot \boldsymbol{\varphi}^{f} d x+\int_{\Gamma^{f p}} \boldsymbol{\sigma}^{p} \boldsymbol{n}^{p} \cdot \boldsymbol{\varphi}^{p} d x+\int_{\Gamma^{f p}}\left(\left.\boldsymbol{v}\right|_{\Gamma^{f p}(t)}-\boldsymbol{V}\right) \cdot \boldsymbol{n}^{f} \psi^{p} d x .
\end{gathered}
$$

Thanks to the conservation of momentum conditions (2.14) and (2.21), the first two terms are equal to zero. Decomposing the stress terms into their normal and tangential components and employing condition (2.18) we have

$$
\begin{gathered}
\mathcal{I}=\left.\int_{\Gamma^{f p}} J \boldsymbol{n}^{f} \cdot \boldsymbol{\sigma}^{f} \boldsymbol{n}^{f}\right|_{\Gamma^{f p}(t)}\left(\left.\boldsymbol{\varphi}^{f}\right|_{\Gamma^{f p}(t)}-\boldsymbol{\varphi}^{p}\right) \cdot \boldsymbol{n}^{f} d x+\int_{\Gamma^{f p}}\left(\left.\boldsymbol{v}\right|_{\Gamma^{f p}(t)}-\boldsymbol{V}\right) \cdot \boldsymbol{n}^{f} \psi^{p} d x \\
\quad+\left.\int_{\Gamma^{f p}} J \boldsymbol{\tau}^{f} \cdot \boldsymbol{\sigma}^{f} \boldsymbol{n}^{f}\right|_{\Gamma^{f p}(t)} P_{\tau}^{f}\left(\left.\boldsymbol{\varphi}^{f}\right|_{\Gamma^{f p}(t)}-\boldsymbol{\varphi}^{p}\right) d x+\int_{\Gamma_{\text {in } / \text { out }}^{f}} \boldsymbol{\sigma}^{f} \boldsymbol{n}^{f} \cdot \boldsymbol{\varphi}^{f} d x .
\end{gathered}
$$

Due to the momentum conservation for the fluid phase (2.17) and the slip condition (2.16), we have

$$
\begin{gathered}
\mathcal{I}=-\int_{\Gamma^{f p}} p_{p}\left(\left.\varphi^{f}\right|_{\Gamma^{f p}(t)}-\varphi^{p}\right) \cdot \boldsymbol{n}^{f} d x+\int_{\Gamma^{f p}}\left(\left.\boldsymbol{v}\right|_{\Gamma^{f p}(t)}-\boldsymbol{V}\right) \cdot \boldsymbol{n}^{f} \psi^{p} d x \\
-\int_{\Gamma^{f p}} \gamma^{f p} P_{\tau}^{f}\left(\left.\boldsymbol{v}\right|_{\Gamma^{f p}(t)}-\boldsymbol{V}\right) P_{\tau}^{f}\left(\left.\varphi^{f}\right|_{\Gamma^{f p}(t)}-\varphi^{p}\right) d x+\int_{\Gamma_{\text {in } / \text { out }}^{f}} \boldsymbol{\sigma}^{f} \boldsymbol{n}^{f} \cdot \boldsymbol{\varphi}^{f} d x .
\end{gathered}
$$

Finally, introducing the bilinear forms defined in (2.30) and (2.31) and employing fluid boundary conditions (2.5)-(2.6), we get

$$
\begin{aligned}
\mathcal{I}= & -c_{p}\left(p_{p},\left.\boldsymbol{\varphi}^{f}\right|_{\Gamma^{f p}(t)}-\boldsymbol{\varphi}^{p}\right)+c_{p}\left(\psi^{p},\left.\boldsymbol{v}\right|_{\Gamma_{f p}(t)}-\boldsymbol{V}\right)-c_{s}\left(\left.\boldsymbol{v}\right|_{\Gamma^{f p}(t)}-\boldsymbol{V},\left.\boldsymbol{\varphi}^{f}\right|_{\Gamma^{f p}(t)}-\boldsymbol{\varphi}^{p}\right) \\
& -\int_{\Gamma_{\text {in }}}\left(p_{\text {in }}(t)-\frac{\rho_{f}}{2}|\boldsymbol{v}|^{2}\right) \boldsymbol{\varphi}^{f} \cdot \boldsymbol{n}^{f} d x-\int_{\Gamma_{\text {out }}}\left(p_{\text {out }}(t)-\frac{\rho_{f}}{2}|\boldsymbol{v}|^{2}\right) \boldsymbol{\varphi}^{f} \cdot \boldsymbol{n}^{f} d x .
\end{aligned}
$$


Thus, the weak formulation of the coupled fluid/structure/poroelastic material system reads as follows: given $t \in(0, T)$ find $\left(\boldsymbol{v}, \boldsymbol{\xi}=\partial_{t} \boldsymbol{\eta}, \boldsymbol{V}=\partial_{t} \boldsymbol{U}, \boldsymbol{q}, p_{f}, p_{p}\right) \in V^{f}(t) \times V^{m} \times V^{p} \times$ $V^{q} \times Q^{f}(t) \times Q^{p}$, with $\boldsymbol{\eta}=\left.\boldsymbol{U}\right|_{\Gamma^{p s}}, \boldsymbol{v} \times \boldsymbol{e}_{z}=0$ on $\Gamma_{\text {in } / \text { out }}^{f}$ and $\left.\boldsymbol{v}\right|_{\Gamma^{f s}(t)}=\boldsymbol{\xi}$, such that for all $\left(\boldsymbol{\varphi}^{f}, \boldsymbol{\zeta}, \boldsymbol{\varphi}^{p}, \boldsymbol{\varphi}^{q}, \psi^{f}, \psi^{p}\right) \in W(t) \times V^{q} \times Q^{f}(t) \times Q^{p}$ with $\varphi^{f} \times \boldsymbol{e}_{z}=0$ on $\Gamma_{\text {in } / \text { out }}^{f}$, we have

$$
\begin{aligned}
& \rho_{f} \int_{\Omega^{f}(t)} \partial_{t} \boldsymbol{v} \cdot \boldsymbol{\varphi}^{f} d \boldsymbol{x}+d_{f}\left(\boldsymbol{v}, \boldsymbol{v}, \boldsymbol{\varphi}^{f}\right)+a_{f}\left(\boldsymbol{v}, \boldsymbol{\varphi}^{f}\right)-b_{f}\left(p_{f}, \boldsymbol{\varphi}^{f}\right)+b_{f}\left(\psi^{f}, \boldsymbol{v}\right)+\rho_{m} h \int_{\Gamma^{s}} \partial_{t} \boldsymbol{\xi} \cdot \boldsymbol{\zeta} d x \\
& +a_{m}(\boldsymbol{\eta}, \boldsymbol{\zeta})+\rho_{b} \int_{\Omega^{p}} \partial_{t} \boldsymbol{V} \cdot \boldsymbol{\varphi}^{p} d \boldsymbol{x}+\rho_{q} \int_{\Omega^{p}} \partial_{t} \boldsymbol{q} \cdot \boldsymbol{\varphi}^{p} d \boldsymbol{x}+a_{e}\left(\boldsymbol{U}, \boldsymbol{\varphi}^{p}\right)-b_{e p}\left(p_{p}, \boldsymbol{\varphi}^{p}\right)+a_{q}\left(\boldsymbol{q}, \boldsymbol{\varphi}^{q}\right) \\
& \quad+b_{q p}\left(p_{p}, \boldsymbol{\varphi}^{q}\right)+\rho_{q} \int_{\Omega^{p}} \partial_{t} \boldsymbol{V} \cdot \boldsymbol{\varphi}^{q} d \boldsymbol{x}+m \int_{\Omega^{p}} \partial_{t} \boldsymbol{q} \cdot \boldsymbol{\varphi}^{q} d \boldsymbol{x}+\int_{\Omega^{p}} \frac{1}{M} \partial_{t} p_{p} \psi^{p} d \boldsymbol{x}+b_{e p}\left(\psi^{p}, \boldsymbol{V}\right) \\
& -b_{q p}\left(\psi^{p}, \boldsymbol{q}\right)+c_{p}\left(p_{p},\left.\boldsymbol{\varphi}^{f}\right|_{\Gamma^{f p}(t)}-\boldsymbol{\varphi}^{p}\right)-c_{p}\left(\psi^{p},\left.\boldsymbol{v}\right|_{\Gamma^{f p}(t)}-\boldsymbol{V}\right)+c_{s}\left(\left.\boldsymbol{v}\right|_{\Gamma^{f p}(t)}-\boldsymbol{V},\left.\boldsymbol{\varphi}^{f}\right|_{\Gamma^{f p}(t)}-\boldsymbol{\varphi}^{p}\right) \\
& \quad=-\int_{\Gamma_{\text {in }}}\left(p_{\text {in }}(t)-\frac{\rho_{f}}{2}|\boldsymbol{v}|^{2}\right) \boldsymbol{\varphi}^{f} \cdot \boldsymbol{n}^{f} d x-\int_{\Gamma_{\text {out }}}\left(p_{\text {out }}(t)-\frac{\rho_{f}}{2}|\boldsymbol{v}|^{2}\right) \boldsymbol{\varphi}^{f} \cdot \boldsymbol{n}^{f} d x .
\end{aligned}
$$

\subsection{Energy estimates for the monolithic problem}

For a domain $\Omega$ we denote by $\|\cdot\|_{H^{k}(\Omega)}$ the norm in the Sobolev space $H^{k}(\Omega)$. We also introduce the following seminorms

$$
\left\|\varphi^{p}\right\|_{E}:=a_{e}\left(\varphi^{p}, \varphi^{p}\right)^{1 / 2}, \quad \forall \varphi^{p} \in V^{p}
$$

and

$$
\|\boldsymbol{\zeta}\|_{M}:=a_{m}(\boldsymbol{\zeta}, \boldsymbol{\zeta})^{1 / 2}, \quad \forall \boldsymbol{\zeta} \in V^{m} .
$$

To derive the energy of the coupled problem let

$$
\left(\boldsymbol{\varphi}^{f}, \boldsymbol{\zeta}, \boldsymbol{\varphi}^{p}, \boldsymbol{\varphi}^{q}, \psi^{f}, \psi^{p}\right)=\left(\boldsymbol{v}, \boldsymbol{\xi}, \boldsymbol{V}, \boldsymbol{q}, p_{f}, p_{p}\right)
$$

in equation (2.38). Derivation of the energy estimates for the Navier-Stokes equations coupled with an elastic structure is standard, some details can be found in $[11,51]$. We denote the terms in the dynamic Biot system that require special attention by $\mathcal{T}$ :

$$
\mathcal{T}=\rho_{b} \int_{\Omega^{p}} \partial_{t} \boldsymbol{V} \cdot \boldsymbol{V} d \boldsymbol{x}+\rho_{q} \int_{\Omega^{p}} \partial_{t} \boldsymbol{q} \cdot \boldsymbol{V} d \boldsymbol{x}+\rho_{q} \int_{\Omega^{p}} \partial_{t} \boldsymbol{V} \cdot \boldsymbol{q} d \boldsymbol{x}+m \int_{\Omega^{p}} \partial_{t} \boldsymbol{q} \cdot \boldsymbol{q} d \boldsymbol{x} .
$$

Note that the terms above can be written as

$$
\mathcal{T}=\frac{\rho_{b}}{2} \frac{d}{d t} \int_{\Omega^{p}}|\boldsymbol{V}|^{2} d \boldsymbol{x}+\rho_{q} \frac{d}{d t} \int_{\Omega^{p}} \boldsymbol{V} \cdot \boldsymbol{q}+\frac{m}{2} \frac{d}{d t} \int_{\Omega^{p}}|\boldsymbol{q}|^{2} d \boldsymbol{x} .
$$

Since $\boldsymbol{q}$ is the average velocity of the fluid relative to the velocity of the solid, namely $\boldsymbol{q}=$ $\phi\left(\boldsymbol{V}_{f}-\boldsymbol{V}\right)$, we have

$$
\mathcal{T}=\frac{\rho_{b}}{2} \frac{d}{d t} \int_{\Omega^{p}}|\boldsymbol{V}|^{2} d \boldsymbol{x}+\rho_{q} \phi \frac{d}{d t} \int_{\Omega^{p}} \boldsymbol{V} \cdot\left(\boldsymbol{V}_{f}-\boldsymbol{V}\right)+\frac{m}{2} \frac{d}{d t} \int_{\Omega^{p}}|\boldsymbol{q}|^{2} d \boldsymbol{x} .
$$

Using the algebraic identity $2(a-b) a=a^{2}-b^{2}+(a-b)^{2}$, we get

$$
\mathcal{T}=\frac{\rho_{b}}{2} \frac{d}{d t}\|\boldsymbol{V}\|_{L^{2}\left(\Omega^{p}\right)}^{2}-\frac{\rho_{q} \phi}{2} \frac{d}{d t}\|\boldsymbol{V}\|_{L^{2}\left(\Omega^{p}\right)}^{2}+\frac{\rho_{q} \phi}{2} \frac{d}{d t}\left\|\boldsymbol{V}_{f}\right\|_{L^{2}\left(\Omega^{p}\right)}^{2}-\frac{\rho_{q} \phi}{2} \frac{d}{d t}\left\|\boldsymbol{V}_{f}-\boldsymbol{V}\right\|_{L^{2}\left(\Omega^{p}\right)}^{2}
$$




$$
+\frac{m}{2} \frac{d}{d t}\|\boldsymbol{q}\|_{L^{2}\left(\Omega^{p}\right)}^{2}
$$

Since $\rho_{b}=(1-\phi) \rho_{s}+\phi \rho_{q}$, and employing the definition of $\boldsymbol{q}$ again, we get

$$
\mathcal{T}=\frac{(1-\phi) \rho_{s}}{2} \frac{d}{d t}\|\boldsymbol{V}\|_{L^{2}\left(\Omega^{p}\right)}^{2}+\frac{\rho_{q} \phi}{2} \frac{d}{d t}\left\|\boldsymbol{V}_{f}\right\|_{L^{2}\left(\Omega^{p}\right)}^{2}-\frac{\rho_{q}}{2 \phi} \frac{d}{d t}\|\boldsymbol{q}\|_{L^{2}\left(\Omega^{p}\right)}^{2}+\frac{m}{2} \frac{d}{d t}\|\boldsymbol{q}\|_{L^{2}\left(\Omega^{p}\right)}^{2} .
$$

Finally, since $m$ is defined as $m=\frac{1}{\phi} \rho_{q}$, terms in $\mathcal{T}$ reduce to the terms due to the kinetic energy of the solid phase and the kinetic energy of the fluid phase:

$$
\mathcal{T}=\frac{(1-\phi) \rho_{s}}{2} \frac{d}{d t}\|\boldsymbol{V}\|_{L^{2}\left(\Omega^{p}\right)}^{2}+\frac{\rho_{q} \phi}{2} \frac{d}{d t}\left\|\boldsymbol{V}_{f}\right\|_{L^{2}\left(\Omega^{p}\right)}^{2}
$$

Thus, the energy equality for coupled system is given as follows:

$$
\frac{d}{d t} \mathcal{E}(t)+\mathcal{D}(t) \leq C\left(\left\|p_{\text {in }}(t)\right\|_{L^{2}\left(\Gamma_{\text {in }}^{f}\right)}^{2}+\left\|p_{\text {out }}(t)\right\|_{L^{2}\left(\Gamma_{\text {out }}^{f}\right)}^{2}\right)
$$

where $\mathcal{E}(t)$ denotes the sum of the kinetic and the elastic energy of the coupled problem

$$
\begin{gathered}
\mathcal{E}(t)=\underbrace{\frac{\rho_{f}}{2}\|\boldsymbol{v}\|_{L^{2}\left(\Omega^{f}(t)\right)}^{2}+\frac{\rho_{m} h}{2}\left\|\partial_{t} \boldsymbol{\eta}\right\|_{L^{2}\left(\Gamma^{s}\right)}^{2}+\frac{(1-\phi) \rho_{s}}{2}\|\boldsymbol{V}\|_{L^{2}\left(\Omega^{p}\right)}^{2}+\frac{\phi \rho_{q}}{2}\left\|\boldsymbol{V}_{f}\right\|_{L^{2}\left(\Omega^{p}\right)}^{2}+\frac{1}{2 M}\left\|p_{p}\right\|_{L^{2}\left(\Omega^{p}\right)}^{2}}_{\text {kinetic energy }} \\
+\underbrace{\frac{1}{2\|\boldsymbol{\eta}\|_{M}^{2}+\frac{1}{2}\|\boldsymbol{U}\|_{E}^{2}},}_{\text {elastic energy }}
\end{gathered}
$$

and $\mathcal{D}(t)$ denotes the dissipative terms

$$
\mathcal{D}(t)=\mu_{f}\|\boldsymbol{D}(\boldsymbol{v})\|_{L^{2}\left(\Omega^{f}(t)\right)}^{2}+\left\|\boldsymbol{\kappa}^{-1} \boldsymbol{q}\right\|_{L^{2}\left(\Omega^{p}\right)}^{2}+\left\|\gamma^{f p} P_{\tau}^{f}\left(\left.\boldsymbol{v}\right|_{\Gamma^{f p}(t)}-\boldsymbol{V}\right)\right\|_{L^{2}\left(\Gamma^{f p}\right)}^{2} .
$$

The constant $C$ that appears on the right-hand side depends only on the coefficients in the problem.

\section{The numerical scheme}

The coupled problem features three separate physical phenomena defined on different domains. Hence, we propose a loosely coupled scheme which consists of three main subproblems: the fluid sub-problem, the structure sub-problem and the poroelasticity subproblem. To split the fluid and structure, we use the kinematically coupled $\beta$-scheme proposed in [11]. The kinematically coupled $\beta$-scheme is a loosely coupled scheme based on the Lie operator splitting. This scheme has been shown to be unconditionally stable, circumventing the problems associated with the added mass effect.

To split the fluid problem from the poroelasticity problem, we modify and extend the domain decomposition approach used in [18] to split the coupled Stokes-Darcy system into Stokes and Darcy sub-problems. This method has been shown to be unconditionally stable and first-order convergent in time. Following this approach, to split the Biot problem from 
the Navier-Stokes problem, we will rewrite the coupling conditions (2.15), (2.16) and (2.17) on $\Gamma^{f p}$, respectively, as follows:

$$
\begin{gathered}
\alpha_{p} p_{p}+(\boldsymbol{V}+\boldsymbol{q}) \cdot \boldsymbol{n}^{f}=\alpha_{p} p_{p}+\left.\boldsymbol{v} \cdot \boldsymbol{n}^{f}\right|_{\Gamma^{f p}(t)}, \\
\alpha_{s} \boldsymbol{V} \cdot \boldsymbol{\tau}_{i}^{f}+\left.J \boldsymbol{\tau}_{i}^{f} \cdot \boldsymbol{\sigma}^{f} \boldsymbol{n}^{f}\right|_{\Gamma^{f p}(t)}=\alpha_{s} \boldsymbol{V} \cdot \boldsymbol{\tau}_{i}^{f}-\gamma^{f p}\left(\left.\boldsymbol{v}\right|_{\Gamma^{f p}(t)}-\boldsymbol{V}\right) \cdot \boldsymbol{\tau}_{i}^{f}, \\
\left.\alpha_{f} \boldsymbol{v} \cdot \boldsymbol{n}^{f}\right|_{\Gamma^{f p}(t)}+\left.J \boldsymbol{n}^{f} \cdot \boldsymbol{\sigma}^{f} \boldsymbol{n}^{f}\right|_{\Gamma^{f p}(t)}=\left.\alpha_{f} \boldsymbol{v} \cdot \boldsymbol{n}^{f}\right|_{\Gamma^{f p}(t)}-p_{p} .
\end{gathered}
$$

Similar to the approach in [8], here we introduce combination parameters $\alpha_{f}, \alpha_{s}$ and $\alpha_{p}$. We assume that $\alpha_{f}, \alpha_{s}, \alpha_{p} \geq 0$ in order for the problem to be well-posed. Combination parameters have been actively used in design of partitioned schemes based on the Robin transmission conditions $[22,17,18,5,6,8]$. In particular, the domain decomposition method presented in [18] corresponds to the case when $\alpha_{f}=1$ and $\alpha_{p}=1$. After the time discretization, the variables multiplying the combination parameters on the left hand side are taken at the current time step, while the values on the right hand side are taken from the previous time step.

Finally, we extend and adapt the operator splitting approach used in [11] to split the thin elastic structure problem from the poroelastic problem. In order to achieve stability, we split the thin structure equation so that the structure inertia is included in both the fluid sub-problem and the poroelasticity sub-problem as a Robin boundary condition. In order to improve the accuracy, following the similar approach as in $[11,16]$, we split the normal fluid stress and the normal poroelastic stress as

$$
\boldsymbol{\sigma}^{f} \boldsymbol{n}^{f}=\underbrace{\boldsymbol{\sigma}^{f} \boldsymbol{n}^{f}+\beta p_{f} \boldsymbol{n}^{f}}_{\text {Part I }} \underbrace{-\beta p_{f} \boldsymbol{n}^{f}}_{\text {Part II }}, \quad \boldsymbol{\sigma}^{p} \boldsymbol{n}^{p}=\underbrace{\boldsymbol{\sigma}^{p} \boldsymbol{n}^{p}+\beta \alpha p_{p} \boldsymbol{n}^{p}}_{\text {Part I }} \underbrace{-\beta \alpha p_{p} \boldsymbol{n}^{p}}_{\text {Part II }} .
$$

Parts II in the equations above will be used as a loading on the thin structure equation.

Let $t^{n}:=n \Delta t$ for $n=1, \ldots, N$, where $T=N \Delta t$ is the final time. We denote by $d_{t}$ the first order discrete time derivative:

$$
d_{t} \phi^{n+1}=\frac{\phi^{n+1}-\phi^{n}}{\Delta t}
$$

The proposed loosely coupled scheme in the time-discrete form is given in the following problems:

P1 (Fluid): Given $t^{n+1} \in(0, T], n=0, \ldots, N-1$ and $\boldsymbol{w}^{n}, \boldsymbol{\xi}^{n}, \boldsymbol{V}^{n}, p_{p}^{n}$ and $p_{f}^{n}$ from the previous time step, find $\boldsymbol{v}^{n+1}$ and $p_{f}^{n+1}$ such that

$$
\begin{array}{lr}
\rho_{f}\left(d_{t} \boldsymbol{v}^{n+1}+\left(\boldsymbol{v}^{n}-\boldsymbol{w}^{n}\right) \cdot \nabla \boldsymbol{v}^{n+1}\right)=\nabla \cdot \boldsymbol{\sigma}^{f}\left(\boldsymbol{v}^{n+1}, p_{f}^{n+1}\right) & \text { in } \Omega^{f}\left(t^{n}\right), \\
\nabla \cdot \boldsymbol{v}^{n+1}=0 & \text { in } \Omega^{f}\left(t^{n}\right), \\
\left.J \boldsymbol{\sigma}^{f} \boldsymbol{n}^{f}\right|_{\Gamma^{f s}(t)}+\left.\frac{\rho_{m} h}{\Delta t} \boldsymbol{v}^{n+1}\right|_{\Gamma^{f s}(t)}=\frac{\rho_{m} h}{\Delta t} \boldsymbol{\xi}^{n}-\left.\beta J p_{f}^{n} \boldsymbol{n}^{f}\right|_{\Gamma^{f s}(t)} & \text { on } \Gamma^{f s}, \\
\left.J \boldsymbol{\tau}^{f} \cdot \boldsymbol{\sigma}^{f} \boldsymbol{n}^{f}\right|_{\Gamma^{f p}(t)}=-\gamma^{f p}\left(\left.\boldsymbol{v}^{n+1}\right|_{\Gamma^{f p}(t)}-\boldsymbol{V}^{n}\right) \cdot \boldsymbol{\tau}_{i}^{f} & i=1,2, \text { on } \Gamma^{f p}, \\
\left.\alpha_{f} \boldsymbol{v}^{n+1} \cdot \boldsymbol{n}^{f}\right|_{\Gamma^{f p}(t)}+\left.J \boldsymbol{n}^{f} \cdot \boldsymbol{\sigma}^{f} \boldsymbol{n}^{f}\right|_{\Gamma^{f p}(t)}=\left.\alpha_{f} \boldsymbol{v}^{n} \cdot \boldsymbol{n}^{f}\right|_{\Gamma^{f p}(t)}-p_{p}^{n} & \text { on } \Gamma^{f p} .
\end{array}
$$


P2 (Poroelasticity): Given $\boldsymbol{v}^{n+1}$ computed in the fluid sub-problem and $\boldsymbol{\xi}^{n}$ computed in the previous time-step, find $\boldsymbol{V}^{n+1}, \boldsymbol{U}^{n+1}, \boldsymbol{q}^{n+1}$, and $p_{p}^{n+1}$ such that

$$
\begin{array}{lr}
\rho_{b} d_{t} \boldsymbol{V}^{n+1}+\rho_{q} d_{t} \boldsymbol{q}^{n+1}=\nabla \cdot \boldsymbol{\sigma}^{p}\left(\boldsymbol{U}^{n+1}, p_{p}^{n+1}\right) & \text { in } \Omega^{p}, \\
d_{t} \boldsymbol{U}^{n+1}=\boldsymbol{V}^{n+1} & \text { in } \Omega^{p}, \\
\boldsymbol{\kappa}^{-1} \boldsymbol{q}^{n+1}=-\nabla p_{p}^{n+1}-\rho_{q} d_{t} \boldsymbol{V}^{n+1}-m d_{t} \boldsymbol{q}^{n+1} & \text { in } \Omega^{p}, \\
\frac{1}{M} d_{t} p_{p}^{n+1}+\alpha \nabla \cdot \boldsymbol{V}^{n+1}+\nabla \cdot \boldsymbol{q}^{n+1}=0 & \text { in } \Omega^{p}, \\
\boldsymbol{\sigma}^{p} \boldsymbol{n}^{p}+\frac{\rho_{m} h}{\Delta t} \boldsymbol{V}^{n+1}=\frac{\rho_{m} h}{\Delta t} \boldsymbol{\xi}^{n}-\beta \alpha p_{p}^{n} \boldsymbol{n}^{p} & \text { on } \Gamma^{p s}, \\
\boldsymbol{q}^{n+1} \cdot \boldsymbol{n}^{p}=0 & (3.5) \\
-\alpha_{s} \boldsymbol{V}^{n+1} \cdot \boldsymbol{\tau}_{i}^{f}+\boldsymbol{\tau}^{f} \cdot \boldsymbol{\sigma}^{p} \boldsymbol{n}^{f}=-\alpha_{s} \boldsymbol{V}^{n} \cdot \boldsymbol{\tau}_{i}^{f}-\gamma^{f p}\left(\left.\boldsymbol{v}^{n+1}\right|_{\Gamma^{f p}(t)}-\boldsymbol{V}^{n+1}\right) \cdot \boldsymbol{\tau}_{i}^{f} & i=1,2, \text { on } \Gamma^{f p}, \\
\boldsymbol{n}^{f} \cdot \boldsymbol{\sigma}^{p} \boldsymbol{n}^{f}=-p_{p}^{n+1} & \text { on } \Gamma^{f p}, \\
\alpha_{p} p_{p}^{n+1}+\boldsymbol{q}^{n+1} \cdot \boldsymbol{n}^{f}=\alpha_{p} p_{p}^{n}+\left(\left.\boldsymbol{v}^{n+1}\right|_{\Gamma^{f p}(t)}-\boldsymbol{V}^{n+1}\right) \cdot \boldsymbol{n}^{f} & \text { on } \Gamma^{f p} .
\end{array}
$$

P3 (Structure): Given $\boldsymbol{v}^{n+1}, p_{f}^{n+1}$ and $\boldsymbol{V}^{n+1}, p_{p}^{n+1}$ computed in (3.4) and (3.5), find $\boldsymbol{\xi}^{n+1}$ and $\boldsymbol{\eta}^{n+1}$ such that

$$
\begin{aligned}
& \rho_{m} h d_{t} \boldsymbol{\xi}^{n+1}+\mathcal{L}\left(\frac{\boldsymbol{\eta}^{n+1}+\boldsymbol{\eta}^{n}}{2}\right)=\beta p^{n+1} \text { on } \Gamma^{s}, \\
& d_{t} \boldsymbol{\eta}^{n+1}=\boldsymbol{\xi}^{n+1} \quad \text { on } \Gamma^{s}, \\
& \boldsymbol{\xi}^{n}= \begin{cases}\left.\boldsymbol{v}^{n}\right|_{\Gamma^{f s}(t)} & \text { on } \Gamma^{f s}, \\
\boldsymbol{V}^{n} & \text { on } \Gamma^{p s},\end{cases}
\end{aligned}
$$

where

$$
p^{n+1}= \begin{cases}\left.J p_{f}^{n+1} \boldsymbol{n}^{f}\right|_{\Gamma^{f s}(t)} & \text { on } \Gamma^{f s}, \\ \alpha p_{p}^{n+1} \boldsymbol{n}^{p} & \text { on } \Gamma^{p s} .\end{cases}
$$

P4 (Geometry): Given the displacement $\boldsymbol{\eta}^{n+1}$ of the boundary $\Gamma^{f s}$ and the displacement $\boldsymbol{U}^{n+1}$ of the boundary $\Gamma^{f p}$, compute the fluid domain $\Omega^{f}\left(t^{n+1}\right)$ and the ALE velocity $\boldsymbol{w}^{n+1}$ as follows:

$$
\mathcal{A}_{t^{n+1}}(\hat{\boldsymbol{x}})=\hat{\boldsymbol{x}}+\operatorname{Ext}\left(\tilde{g}^{n+1}\right) \quad \forall \hat{\boldsymbol{x}} \in \Omega^{f}, \quad \Omega^{f}\left(t^{n+1}\right)=\mathcal{A}_{t^{n+1}}\left(\Omega^{f}\right), \quad \boldsymbol{w}^{n+1}=d_{t} \boldsymbol{x}^{n+1},
$$

where $\boldsymbol{x}^{n+1} \in \Omega^{f}\left(t^{n+1}\right), \boldsymbol{x}^{n} \in \Omega^{f}\left(t^{n}\right)$ and

$$
\tilde{g}^{n+1}= \begin{cases}\boldsymbol{\eta}^{n+1} & \text { on } \Gamma^{f s}, \\ \boldsymbol{U}^{n+1} & \text { on } \Gamma^{f p} \\ \mathbf{0} & \text { on } \Gamma_{\text {in } / \text { out }}^{f}\end{cases}
$$

Remark 2. The proposed algorithm can be easily extended to the case when the vessel wall is modeled using a composite structure model consisting of a thin elastic layer and a thick elastic 
layer. In that case, the thin structure sub-problem can be treated as a Robin condition for the thick structure sub-problem as in [12]. However, the extension of the proposed algorithm to the case when the vessel wall is modeled using a single thick elastic structure is not straightforward and is out of scope of this paper.

\section{Weak formulation and stability}

For simplicity, we analyze the problem assuming the time-dependent Stokes model for the fluid flow driven by the boundary conditions (2.3)-(2.4). We also assume that the displacement of the boundary is small enough and can be neglected. Under these assumptions, domain $\Omega^{f}(t)$ is fixed:

$$
\Omega^{f}(t)=\Omega^{f}, \quad \forall t \in(0, T) .
$$

This assumption is common in FSI modeling [20, 14, 28, 16]. Although simplified, this problem still retains the main difficulties associated with the "added mass" effect and the difficulties that partitioned schemes encounter when modeling interaction between the fluid flow and the poroelastic material.

\subsection{Weak formulation of the simplified, fully-discrete problem}

Let the test function spaces $V^{f}, Q^{f}, V^{m}, V^{p} . V^{q}$ and $Q^{p}$ be defined as in Section 2.4. To discretize the problem in space, we use the finite element method. Thus, we define the finite element spaces $V_{h}^{f} \subset V^{f}, Q_{h}^{f} \subset Q^{f}, V_{h}^{p} \subset V^{p}, V_{h}^{q} \subset V^{q}, Q_{h}^{p} \subset Q^{p}$, and $V_{h}^{m} \subset V^{m}$ based on a conforming finite element triangulation with maximum triangle diameter $\Delta x$. We assume that spaces $V_{h}^{f}$ and $Q_{h}^{f}$ are inf-sup stable. The fully discrete numerical scheme in the weak formulation is given as follows:

P1d (Fluid): Given $t^{n+1} \in(0, T], n=0, \ldots, N-1$ and $\boldsymbol{\xi}_{h}^{n}, \boldsymbol{V}_{h}^{n}, p_{p, h}^{n}$ and $p_{h, f}^{n}$ from the previous time step, find $\boldsymbol{v}_{h}^{n+1} \in V^{f}$ and $p_{f, h}^{n+1} \in Q^{f}$ such that for all $\left(\boldsymbol{\varphi}_{h}^{f}, \psi_{h}^{f}\right) \in V_{h}^{f} \times Q_{h}^{f}$

$$
\begin{gathered}
\rho_{f} \int_{\Omega^{f}} d_{t} \boldsymbol{v}_{h}^{n+1} \cdot \boldsymbol{\varphi}_{h}^{f} d \boldsymbol{x}+a_{f}\left(\boldsymbol{v}_{h}^{n+1}, \boldsymbol{\varphi}_{h}^{f}\right)-b_{f}\left(p_{f, h}^{n+1}, \boldsymbol{\varphi}_{h}^{f}\right)+b_{f}\left(\psi_{h}^{f}, \boldsymbol{v}_{h}^{n+1}\right)+\frac{\rho_{m} h}{\Delta t} \int_{\Gamma^{f s}} \boldsymbol{v}_{h}^{n+1} \cdot \boldsymbol{\varphi}_{h}^{f} d x \\
+\alpha_{f} \int_{\Gamma^{f p}}\left(\boldsymbol{v}_{h}^{n+1} \cdot \boldsymbol{n}^{f}\right)\left(\boldsymbol{\varphi}_{h}^{f} \cdot \boldsymbol{n}^{f}\right) d x=\frac{\rho_{m} h}{\Delta t} \int_{\Gamma^{f s}} \boldsymbol{\xi}_{h}^{n} \cdot \boldsymbol{\varphi}_{h}^{f} d x+\alpha_{f} \int_{\Gamma^{f p}}\left(\boldsymbol{v}_{h}^{n} \cdot \boldsymbol{n}^{f}\right)\left(\boldsymbol{\varphi}_{h}^{f} \cdot \boldsymbol{n}^{f}\right) d x \\
-\beta \int_{\Gamma^{f s}} p_{f, h}^{n} \boldsymbol{n}^{f} \cdot \boldsymbol{\varphi}_{h}^{f} d x-c_{s}\left(\boldsymbol{v}_{h}^{n+1}-\boldsymbol{V}_{h}^{n}, \varphi_{h}^{f}\right)-c_{p}\left(p_{p, h}^{n}, \boldsymbol{\varphi}_{h}^{f}\right)-\int_{\Gamma_{\text {in }}} p_{\text {in }}\left(t^{n+1}\right) \boldsymbol{\varphi}_{h}^{f} \cdot \boldsymbol{n}^{f} d x \\
-\int_{\Gamma_{\text {out }}} p_{\text {out }}\left(t^{n+1}\right) \boldsymbol{\varphi}_{h}^{f} \cdot \boldsymbol{n}^{f} d x .
\end{gathered}
$$

P2d (Poroelasticity): Given $\boldsymbol{v}_{h}^{n+1}$ computed in (4.1) and $\boldsymbol{\xi}_{h}^{n}$ and $p_{p, h}^{n}$ computed in the previous time-step, find $\boldsymbol{V}_{h}^{n+1} \in V_{h}^{p}, \boldsymbol{q}_{h}^{n+1} \in V_{h}^{q}$, and $p_{p, h}^{n+1} \in Q_{h}^{p}$ such that for all $\left(\varphi_{h}^{f}, \psi_{h}^{f}\right) \in$ $V_{h}^{f} \times Q_{h}^{f}$

$$
\begin{gathered}
\rho_{b} \int_{\Omega^{p}} d_{t} \boldsymbol{V}_{h}^{n+1} \cdot \boldsymbol{\varphi}_{h}^{p} d \boldsymbol{x}+\rho_{q} \int_{\Omega^{p}} d_{t} \boldsymbol{q}_{h}^{n+1} \cdot \boldsymbol{\varphi}_{h}^{p} d \boldsymbol{x}+a_{e}\left(\boldsymbol{U}_{h}^{n+1}, \boldsymbol{\varphi}_{h}^{p}\right)-b_{e p}\left(p_{p, h}^{n+1}, \boldsymbol{\varphi}_{h}^{p}\right)+a_{q}\left(\boldsymbol{q}_{h}^{n+1}, \boldsymbol{\varphi}_{h}^{q}\right) \\
\quad+b_{q p}\left(p_{p, h}^{n+1}, \boldsymbol{\varphi}_{h}^{q}\right)+\rho_{q} \int_{\Omega^{p}} d_{t} \boldsymbol{V}_{h}^{n+1} \cdot \boldsymbol{\varphi}_{h}^{q} d \boldsymbol{x}+m \int_{\Omega^{p}} d_{t} \boldsymbol{q}_{h}^{n+1} \cdot \boldsymbol{\varphi}_{h}^{q} d \boldsymbol{x}+\int_{\Omega^{p}} \frac{1}{M} d_{t} p_{p, h}^{n+1} \psi_{h}^{p} d \boldsymbol{x}
\end{gathered}
$$




$$
\begin{aligned}
& +b_{e p}\left(\psi_{h}^{p}, \boldsymbol{V}_{h}^{n+1}\right)-b_{q p}\left(\psi_{h}^{p}, \boldsymbol{q}_{h}^{n+1}\right)+\frac{\rho_{m} h}{\Delta t} \int_{\Gamma^{p s}} \boldsymbol{V}_{h}^{n+1} \cdot \boldsymbol{\varphi}_{h}^{p} d x+\alpha_{s} \int_{\Gamma^{f p}}\left(\boldsymbol{V}_{h}^{n+1} \cdot \boldsymbol{\tau}^{f}\right)\left(\boldsymbol{\varphi}_{h}^{p} \cdot \boldsymbol{\tau}^{f}\right) d x \\
& +\alpha_{p} \int_{\Gamma^{f p}} p_{p, h}^{n+1} \psi_{h}^{p} d x=\frac{\rho_{m} h}{\Delta t} \int_{\Gamma^{p s}} \boldsymbol{\xi}_{h}^{n} \cdot \boldsymbol{\varphi}_{h}^{p} d x+\alpha_{s} \int_{\Gamma^{f p}}\left(\boldsymbol{V}_{h}^{n} \cdot \boldsymbol{\tau}^{f}\right)\left(\boldsymbol{\varphi}_{h}^{p} \cdot \boldsymbol{\tau}^{f}\right) d x+\alpha_{p} \int_{\Gamma^{f p}} p_{p, h}^{n} \psi_{h}^{p} d x \\
& \quad-\beta \alpha \int_{\Gamma^{p s}} p_{p, h}^{n} \boldsymbol{n}^{p} \cdot \boldsymbol{\varphi}_{h}^{p} d x+c_{p}\left(p_{p, h}^{n+1}, \boldsymbol{\varphi}_{h}^{p}\right)+c_{s}\left(\boldsymbol{v}_{h}^{n+1}-\boldsymbol{V}_{h}^{n+1}, \boldsymbol{\varphi}_{h}^{p}\right)+c_{p}\left(\psi_{h}^{p}, \boldsymbol{v}_{h}^{n+1}-\boldsymbol{V}_{h}^{n+1}\right) .
\end{aligned}
$$

P3d (Structure): Given $\boldsymbol{v}_{h}^{n+1}, p_{f, h}^{n+1}$ and $\boldsymbol{V}_{h}^{n+1}, p_{p, h}^{n+1}$ computed in (4.1) and (4.2), find $\boldsymbol{\xi}_{h}^{n+1} \in V_{h}^{m}$ such that for all $\boldsymbol{\zeta}_{h} \in V_{h}^{m}$

$$
\begin{gathered}
\frac{\rho_{m} h}{\Delta t} \int_{\Gamma^{s}} \boldsymbol{\xi}_{h}^{n+1} \cdot \boldsymbol{\zeta}_{h} d x+ \\
a_{m}\left(\left(\boldsymbol{\eta}_{h}^{n+1}+\boldsymbol{\eta}_{h}^{n}\right) / 2, \boldsymbol{\zeta}_{h}\right)=\frac{\rho_{m} h}{\Delta t} \int_{\Gamma^{f s}} \boldsymbol{v}_{h}^{n+1} \cdot \boldsymbol{\zeta}_{h} d x+\frac{\rho_{m} h}{\Delta t} \int_{\Gamma^{p s}} \boldsymbol{V}_{h}^{n+1} \cdot \boldsymbol{\zeta}_{h} d x \\
+\beta \int_{\Gamma^{f s}} p_{f, h}^{n+1} \boldsymbol{n}^{f} \cdot \boldsymbol{\zeta}_{h} d x+\beta \alpha \int_{\Gamma^{p s}} p_{p, h}^{n+1} \boldsymbol{n}^{p} \cdot \boldsymbol{\zeta}_{h} d x
\end{gathered}
$$

Remark 3. In problems (4.2) and (4.3), structural displacements $\boldsymbol{U}_{h}^{n+1}$ and $\boldsymbol{\eta}_{h}^{n+1}$ can be eliminated by employing, respectively,

$$
\boldsymbol{U}_{h}^{n+1}=\Delta t \boldsymbol{V}_{h}^{n+1}+\boldsymbol{U}_{h}^{n} \quad \text { and } \quad \boldsymbol{\eta}_{h}^{n+1}=\Delta t \boldsymbol{\xi}_{h}^{n+1}+\boldsymbol{\eta}_{h}^{n} .
$$

After the displacement velocities $\boldsymbol{V}_{h}^{n+1}$ and $\boldsymbol{\xi}_{h}^{n+1}$ are computed, equations above can be used to compute the displacements.

In order to derive the energy estimates and analyze the stability of system (4.1)-(4.3), we assume that $\beta=0$. Cases $\beta=0$ and $\beta=1$ are considered in Section 5. Our previous findings related to FSI problems indicate that taking $\beta>0$ improves the accuracy, without affecting the stability. Moreover, unconditional stability of the kinematically coupled $\beta$-scheme for $\beta \in[0,1]$ was analytically shown in [16] on a simplified FSI problem.

\subsection{Stability analysis for $\beta=0$}

Let $\mathcal{E}^{n}=\mathcal{E}_{f}^{n}+\mathcal{E}_{m}^{n}+\mathcal{E}_{p}^{n}$ denote the total discrete energy of the system (4.1)-(4.3) at time level $n$, where $\mathcal{E}_{f}^{n}$ denotes the discrete energy of the fluid problem, $\mathcal{E}_{m}^{n}$ denotes the discrete energy of the thin elastic structure and $\mathcal{E}_{p}^{n}$ denotes the discrete energy of the Biot problem, defined respectively by

$$
\begin{aligned}
\mathcal{E}_{f}^{n}= & \frac{\rho_{f}}{2}\left\|\boldsymbol{v}_{h}^{n}\right\|_{L^{2}\left(\Omega^{f}\right)}^{2}+\frac{\Delta t \alpha_{f}}{2}\left\|\boldsymbol{v}_{h}^{n} \cdot \boldsymbol{n}^{f}\right\|_{L^{2}\left(\Gamma^{f p}\right)}^{2}, \\
\mathcal{E}_{m}^{n}= & \frac{\rho_{m} h}{2}\left\|\boldsymbol{\xi}_{h}^{n}\right\|_{L^{2}\left(\Gamma^{s}\right)}^{2}+\frac{1}{2}\left\|\boldsymbol{\eta}_{h}^{n}\right\|_{M}^{2}, \\
\mathcal{E}_{p}^{n}= & \frac{(1-\phi) \rho_{s}}{2}\left\|\boldsymbol{V}_{h}^{n}\right\|_{L^{2}\left(\Omega^{p}\right)}^{2}+\frac{\phi \rho_{q}}{2}\left\|\boldsymbol{V}_{f, h}^{n}\right\|_{L^{2}\left(\Omega^{p}\right)}^{2}+\frac{1}{2 M}\left\|p_{p, h}^{n}\right\|_{L^{2}\left(\Omega^{p}\right)}^{2}+\frac{1}{2}\left\|\boldsymbol{U}_{h}^{n}\right\|_{E}^{2} \\
& +\frac{\Delta t \alpha_{s}}{2}\left\|\boldsymbol{V}_{h}^{n} \cdot \boldsymbol{\tau}^{f}\right\|_{L^{2}\left(\Gamma^{f p}\right)}^{2}+\frac{\Delta t \alpha_{p}}{2}\left\|p_{p, h}^{n}\right\|_{L^{2}\left(\Gamma^{f p}\right) .}^{2}
\end{aligned}
$$

The stability of the loosely-coupled scheme (4.1)-(4.3) is stated in the following result. 
Theorem 1. Let $\left\{\left(\boldsymbol{v}_{h}^{n}, \boldsymbol{\xi}_{h}^{n}, \boldsymbol{V}_{h}^{n}, \boldsymbol{q}_{h}^{n}, p_{f, h}^{n}, p_{p, h}^{n}\right)\right\}_{0 \leq n \leq N}$ be the solution of (4.1)-(4.3). Then, under conditions

$$
\alpha_{s}>\frac{2\left(\gamma^{f p}\right)^{2} C_{T}^{2} C_{K}^{2} C_{P}}{\mu_{f}} \quad \alpha_{p}>\frac{2 C_{T}^{2} C_{K}^{2} C_{P}}{\mu_{f}},
$$

the scheme (4.1)-(4.3) is stable and the following estimate holds:

$$
\mathcal{E}^{n+1}+\Delta t \mathcal{D}^{n+1}+\Delta t \mathcal{N}^{n+1} \leq \mathcal{E}^{n}+C \Delta t\left(\left\|p_{\text {in }}\left(t^{n}\right)\right\|_{L^{2}\left(\Gamma_{\text {in }}^{f}\right)}^{2}+\left\|p_{\text {out }}\left(t^{n}\right)\right\|_{L^{2}\left(\Gamma_{\text {out }}^{f}\right)}^{2}\right),
$$

where $\mathcal{D}^{n+1}$ denotes the dissipative terms

$$
\mathcal{D}^{n+1}=\mu_{f}\left\|\boldsymbol{D}\left(\boldsymbol{v}_{h}^{n+1}\right)\right\|_{L^{2}\left(\Omega^{f}\right)}^{2}+\left\|\boldsymbol{\kappa}^{-1} \boldsymbol{q}_{h}^{n+1}\right\|_{L^{2}\left(\Omega^{p}\right)}^{2}+\gamma^{f p}\left\|P_{\tau}^{f}\left(\left.\boldsymbol{v}_{h}^{n+1}\right|_{\Gamma^{f p}}-\boldsymbol{V}_{h}^{n+1}\right)\right\|_{L^{2}\left(\Gamma^{f p}\right)}^{2},
$$

$\mathcal{N}^{n+1}$ denotes terms due to the numerical dissipation

$$
\begin{gathered}
\mathcal{N}^{n+1}=\frac{\rho_{f} \Delta t}{2}\left\|d_{t} \boldsymbol{v}_{h}^{n+1}\right\|_{L^{2}\left(\Omega^{f}\right)}^{2}+\frac{(1-\phi) \rho_{s} \Delta t}{4}\left\|d_{t} \boldsymbol{V}_{h}^{n+1}\right\|_{L^{2}\left(\Omega^{p}\right)}^{2}+\frac{\phi \rho_{q} \Delta t}{2}\left\|d_{t} \boldsymbol{V}_{f, h}^{n+1}\right\|_{L^{2}\left(\Omega^{p}\right)}^{2} \\
+\frac{\Delta t}{2}\left\|d_{t} \boldsymbol{U}_{h}^{n+1}\right\|_{E}^{2}+\frac{\Delta t}{4 M}\left\|d_{t} p_{p, h}^{n+1}\right\|_{L^{2}\left(\Omega^{p}\right)}^{2}+\frac{\rho_{m} h \Delta t}{2}\left\|d_{t} \boldsymbol{\xi}_{h}^{n+1}\right\|_{L^{2}\left(\Gamma^{s}\right)}^{2}+\frac{\alpha_{f}}{2}\left\|\left(\boldsymbol{v}_{h}^{n+1}-\boldsymbol{v}_{h}^{n}\right) \cdot \boldsymbol{n}^{f}\right\|_{L^{2}\left(\Gamma^{f p}\right)}^{2} \\
+\left(\frac{\alpha_{s}}{2}-\frac{\left(\gamma^{f p}\right)^{2} C_{T}^{2} C_{K}^{2} C_{P}}{\mu_{f}}\right)\left\|\left(\boldsymbol{V}_{h}^{n+1}-\boldsymbol{V}_{h}^{n}\right) \cdot \boldsymbol{\tau}^{f}\right\|_{L^{2}\left(\Gamma^{f p}\right)}^{2} \\
+\left(\frac{\alpha_{p}}{2}-\frac{C_{T}^{2} C_{K}^{2} C_{P}}{\mu_{f}}\right)\left\|\left(p_{p, h}^{n+1}-p_{p, h}^{n}\right)\right\|_{L^{2}\left(\Gamma^{f p}\right)}^{2},
\end{gathered}
$$

and $C$ is a constant that does not depend on the space and time discretization parameters.

Proof. To prove the energy estimate, we test the problem $(4.1)$ with $\left(\varphi_{h}^{f}, \psi_{h}^{f}\right)=\left(\boldsymbol{v}_{h}^{n+1}, p_{f, h}^{n+1}\right)$, problem (4.2) with $\left(\boldsymbol{\varphi}_{h}^{p}, \boldsymbol{\varphi}_{h}^{q}, \psi_{h}^{p}\right)=\left(d_{t} \boldsymbol{U}_{h}^{n+1}=\boldsymbol{V}_{h}^{n+1}, \boldsymbol{q}_{h}^{n+1}, p_{p, h}^{n+1}\right)$ and problem (4.3) with $\boldsymbol{\zeta}_{h}=\boldsymbol{\xi}_{h}^{n+1}=d_{t} \boldsymbol{\eta}_{h}^{n+1}$. Adding the equations together and multiplying by $\Delta t$ we get

$$
\begin{gathered}
\frac{\rho_{f}}{2}\left(\left\|\boldsymbol{v}_{h}^{n+1}\right\|_{L^{2}\left(\Omega^{f}\right)}^{2}-\left\|\boldsymbol{v}_{h}^{n}\right\|_{L^{2}\left(\Omega^{f}\right)}^{2}+\left\|\boldsymbol{v}_{h}^{n+1}-\boldsymbol{v}_{h}^{n}\right\|_{L^{2}\left(\Omega^{f}\right)}^{2}\right)+2 \mu_{f} \Delta t\left\|\boldsymbol{D}\left(\boldsymbol{v}_{h}^{n+1}\right)\right\|_{L^{2}\left(\Omega^{f}\right)}^{2} \\
+\frac{\rho_{m} h}{2}\left(\left\|\boldsymbol{v}_{h}^{n+1}\right\|_{L^{2}\left(\Gamma^{f s}\right)}^{2}-\left\|\boldsymbol{\xi}_{h}^{n}\right\|_{L^{2}\left(\Gamma^{f s}\right)}^{2}+\left\|\boldsymbol{v}_{h}^{n+1}-\boldsymbol{\xi}_{h}^{n}\right\|_{L^{2}\left(\Gamma^{f s}\right)}^{2}\right) \\
+\frac{\alpha_{f} \Delta t}{2}\left(\left\|\boldsymbol{v}_{h}^{n+1} \cdot \boldsymbol{n}^{f}\right\|_{L^{2}\left(\Gamma^{f p}\right)}^{2}-\left\|\boldsymbol{v}_{h}^{n} \cdot \boldsymbol{n}^{f}\right\|_{L^{2}\left(\Gamma^{f p}\right)}^{2}+\left\|\left(\boldsymbol{v}_{h}^{n+1}-\boldsymbol{v}_{h}^{n}\right) \cdot \boldsymbol{n}^{f}\right\|_{L^{2}\left(\Gamma^{f p}\right)}^{2}\right) \\
+\frac{\rho_{b}}{2}\left(\left\|\boldsymbol{V}_{h}^{n+1}\right\|_{L^{2}\left(\Omega^{p}\right)}^{2}-\left\|\boldsymbol{V}_{h}^{n}\right\|_{L^{2}\left(\Omega^{p}\right)}^{2}+\left\|\boldsymbol{V}_{h}^{n+1}-\boldsymbol{V}_{h}^{n}\right\|_{L^{2}\left(\Omega^{p}\right)}^{2}\right)+\rho_{q} \Delta t \int_{\Omega^{p}} d_{t} \boldsymbol{q}_{h}^{n+1} \cdot \boldsymbol{V}_{h}^{n+1} d \boldsymbol{x} \\
+\frac{1}{2}\left(\left\|\boldsymbol{U}_{h}^{n+1}\right\|_{E}^{2}-\left\|\boldsymbol{U}_{h}^{n}\right\|_{E}^{2}+\left\|\boldsymbol{U}_{h}^{n+1}-\boldsymbol{U}_{h}^{n}\right\|_{E}^{2}\right)+\Delta t\left\|\boldsymbol{\kappa}^{-1} \boldsymbol{q}_{h}^{n+1}\right\|_{L^{2}\left(\Omega^{p}\right)}^{2}+\rho_{q} \Delta t \int_{\Omega^{p}} d_{t} \boldsymbol{V}_{h}^{n+1} \cdot \boldsymbol{q}_{h}^{n+1} d \boldsymbol{x} \\
+\frac{m}{2}\left(\left\|\boldsymbol{q}_{h}^{n+1}\right\|_{L^{2}\left(\Omega^{p}\right)}^{2}-\left\|\boldsymbol{q}_{h}^{n}\right\|_{L^{2}\left(\Omega^{p}\right)}^{2}+\left\|\boldsymbol{q}_{h}^{n+1}-\boldsymbol{q}_{h}^{n}\right\|_{L^{2}\left(\Omega^{p}\right)}^{2}\right)+\frac{1}{2 M}\left(\left\|p_{p, h}^{n+1}\right\|_{L^{2}\left(\Omega^{p}\right)}^{2}-\left\|p_{p, h}^{n}\right\|_{L^{2}\left(\Omega^{p}\right)}^{2}\right. \\
\left.+\left\|p_{p, h}^{n+1}-p_{p, h}^{n}\right\|_{L^{2}\left(\Omega^{p}\right)}^{2}\right)+\frac{\rho_{m} h}{2}\left(\left\|\boldsymbol{V}_{h}^{n+1}\right\|_{L^{2}\left(\Gamma^{p s}\right)}^{2}-\left\|\boldsymbol{\xi}_{h}^{n}\right\|_{L^{2}\left(\Gamma^{p s}\right)}^{2}+\left\|\boldsymbol{V}_{h}^{n+1}-\boldsymbol{\xi}_{h}^{n}\right\|_{L^{2}\left(\Gamma^{p s}\right)}^{2}\right) \\
+\frac{\alpha_{s} \Delta t}{2}\left(\left\|\boldsymbol{V}_{h}^{n+1} \cdot \boldsymbol{\tau}^{f}\right\|_{L^{2}\left(\Gamma^{f p}\right)}^{2}-\left\|\boldsymbol{V}_{h}^{n} \cdot \boldsymbol{\tau}^{f}\right\|_{L^{2}\left(\Gamma^{f p}\right)}^{2}+\left\|\left(\boldsymbol{V}_{h}^{n+1}-\boldsymbol{V}_{h}^{n}\right) \cdot \boldsymbol{\tau}^{f}\right\|_{L^{2}\left(\Gamma^{f p}\right)}^{2}\right) \\
+\frac{\rho_{m} h}{2}\left(\left\|\boldsymbol{\xi}_{h}^{n+1}\right\|_{L^{2}\left(\Gamma^{s}\right)}^{2}-\left\|\boldsymbol{v}_{h}^{n+1}\right\|_{L^{2}\left(\Gamma^{f s}\right)}^{2}-\left\|\boldsymbol{V}_{h}^{n+1}\right\|_{L^{2}\left(\Gamma^{p s}\right)}^{2}+\left\|\boldsymbol{\xi}_{h}^{n+1}-\boldsymbol{v}_{h}^{n+1}\right\|_{L^{2}\left(\Gamma^{f s}\right)}^{2}\right.
\end{gathered}
$$




$$
\begin{gathered}
\left.+\left\|\boldsymbol{\xi}_{h}^{n+1}-\boldsymbol{V}_{h}^{n+1}\right\|_{L^{2}\left(\Gamma^{p s}\right)}^{2}\right)+\frac{\alpha_{p} \Delta t}{2}\left(\left\|p_{p, h}^{n+1}\right\|_{L^{2}\left(\Gamma^{f p}\right)}^{2}-\left\|p_{p, h}^{n}\right\|_{L^{2}\left(\Gamma^{f p}\right)}^{2}+\left\|p_{p, h}^{n+1}-p_{p, h}^{n}\right\|_{L^{2}\left(\Gamma^{f p}\right)}^{2}\right) \\
+\frac{1}{2}\left(\left\|\boldsymbol{\eta}_{h}^{n+1}\right\|_{M}^{2}-\left\|\boldsymbol{\eta}_{h}^{n}\right\|_{M}^{2}\right)=-\Delta t c_{s}\left(\boldsymbol{v}_{h}^{n+1}-\boldsymbol{V}_{h}^{n}, \boldsymbol{v}_{h}^{n+1}\right)+\Delta t c_{s}\left(\boldsymbol{v}_{h}^{n+1}-\boldsymbol{V}_{h}^{n+1}, \boldsymbol{V}_{h}^{n+1}\right) \\
-\Delta t c_{p}\left(p_{p, h}^{n}, \boldsymbol{v}_{h}^{n+1}\right)+\Delta t c_{p}\left(p_{p, h}^{n+1}, \boldsymbol{v}_{h}^{n+1}\right)-\Delta t \int_{\Gamma_{i n}^{f}} p_{i n}\left(t^{n+1}\right) \boldsymbol{v}_{h}^{n+1} \cdot \boldsymbol{n}^{f} d x \\
-\Delta t \int_{\Gamma_{\text {out }}^{f}} p_{\text {out }}\left(t^{n+1}\right) \boldsymbol{v}_{h}^{n+1} \cdot \boldsymbol{n}^{f} d x
\end{gathered}
$$

Notice that we can cancel $-\left\|\boldsymbol{v}_{h}^{n+1}\right\|_{L^{2}\left(\Gamma^{f s}\right)}^{2}$ and $\left\|\boldsymbol{v}_{h}^{n+1}\right\|_{L^{2}\left(\Gamma^{f s}\right)}^{2}$, as well as $-\left\|\boldsymbol{V}_{h}^{n+1}\right\|_{L^{2}\left(\Gamma^{p s}\right)}^{2}$ and $\left\|\boldsymbol{V}_{h}^{n+1}\right\|_{L^{2}\left(\Gamma^{p s}\right)}^{2}$. To deal with $\mathcal{T}_{h}^{n+1}=\rho_{q} \Delta t \int_{\Omega^{p}} d_{t} \boldsymbol{q}_{h}^{n+1} \cdot \boldsymbol{V}_{h}^{n+1} d \boldsymbol{x}+\rho_{q} \Delta t \int_{\Omega^{p}} d_{t} \boldsymbol{V}_{h}^{n+1} \cdot \boldsymbol{q}_{h}^{n+1} d \boldsymbol{x}$, similarly as in the continuous case, we employ the discrete product rule

$$
\boldsymbol{V}_{h}^{n+1} d_{t} \boldsymbol{q}_{h}^{n+1}+\boldsymbol{q}_{h}^{n} d_{t} \boldsymbol{V}_{h}^{n+1}=d_{t}\left(\boldsymbol{V}_{h}^{n+1} \boldsymbol{q}_{h}^{n+1}\right)
$$

Now we have

$$
\mathcal{T}_{h}^{n+1}=\rho_{q} \Delta t \int_{\Omega^{p}}\left(d_{t}\left(\boldsymbol{V}_{h}^{n+1} \boldsymbol{q}_{h}^{n+1}\right)+\left(\boldsymbol{q}_{h}^{n+1}-\boldsymbol{q}_{h}^{n}\right) \cdot d_{t} \boldsymbol{V}_{h}^{n+1}\right) d \boldsymbol{x} .
$$

Using $\boldsymbol{q}_{h}^{n+1}=\phi\left(\boldsymbol{V}_{f, h}^{n+1}-\boldsymbol{V}_{h}^{n+1}\right)$ in the first term of (4.8), we get

$$
\begin{gathered}
\rho_{q} \Delta t \int_{\Omega^{p}} d_{t}\left(\boldsymbol{V}_{h}^{n+1} \boldsymbol{q}_{h}^{n+1}\right) d \boldsymbol{x}=\rho_{q} \phi \int_{\Omega^{p}}\left(\boldsymbol{V}_{f, h}^{n+1}-\boldsymbol{V}_{h}^{n+1}\right) \cdot \boldsymbol{V}_{h}^{n+1} d \boldsymbol{x}-\rho_{q} \phi \int_{\Omega^{p}}\left(\boldsymbol{V}_{f, h}^{n}-\boldsymbol{V}_{h}^{n}\right) \cdot \boldsymbol{V}_{h}^{n} d \boldsymbol{x} \\
=-\frac{\rho_{q} \phi}{2}\left\|\boldsymbol{V}_{h}^{n+1}\right\|_{L^{2}\left(\Omega^{p}\right)}^{2}+\frac{\rho_{q} \phi}{2}\left\|\boldsymbol{V}_{f, h}^{n+1}\right\|_{L^{2}\left(\Omega^{p}\right)}^{2}-\frac{\rho_{q} \phi}{2}\left\|\boldsymbol{V}_{f, h}^{n+1}-\boldsymbol{V}^{n+1}\right\|_{L^{2}\left(\Omega^{p}\right)}^{2}+\frac{\rho_{q} \phi}{2}\left\|\boldsymbol{V}_{h}^{n}\right\|_{L^{2}\left(\Omega^{p}\right)}^{2} \\
-\frac{\rho_{q} \phi}{2}\left\|\boldsymbol{V}_{f, h}^{n}\right\|_{L^{2}\left(\Omega^{p}\right)}^{2}+\frac{\rho_{q} \phi}{2}\left\|\boldsymbol{V}_{f, h}^{n}-\boldsymbol{V}_{h}^{n}\right\|_{L^{2}\left(\Omega^{p}\right)}^{2}=\frac{\rho_{q} \phi}{2}\left\|\boldsymbol{V}_{f, h}^{n+1}\right\|_{L^{2}\left(\Omega^{p}\right)}^{2}-\frac{\rho_{q} \phi}{2}\left\|\boldsymbol{V}_{f, h}^{n}\right\|_{L^{2}\left(\Omega^{p}\right)}^{2} \\
-\frac{\rho_{q} \phi}{2}\left\|\boldsymbol{V}_{h}^{n+1}\right\|_{L^{2}\left(\Omega^{p}\right)}^{2}+\frac{\rho_{q} \phi}{2}\left\|\boldsymbol{V}_{h}^{n}\right\|_{L^{2}\left(\Omega^{p}\right)}^{2}-\frac{m}{2}\left\|\boldsymbol{q}_{h}^{n+1}\right\|_{L^{2}\left(\Omega^{p}\right)}^{2}+\frac{m}{2}\left\|\boldsymbol{q}_{h}^{n}\right\|_{L^{2}\left(\Omega^{p}\right)}^{2} .
\end{gathered}
$$

Using $\boldsymbol{q}_{h}^{n+1}=\phi\left(\boldsymbol{V}_{f, h}^{n+1}-\boldsymbol{V}_{h}^{n+1}\right)$ in the second term of (4.8), we get

$$
\begin{aligned}
& \rho_{q} \Delta t \int_{\Omega^{p}}\left(\boldsymbol{q}_{h}^{n+1}-\boldsymbol{q}_{h}^{n}\right) \cdot d_{t} \boldsymbol{V}_{h}^{n+1} d \boldsymbol{x}=-\rho_{q} \phi \Delta t^{2} \int_{\Omega^{p}}\left(d_{t} \boldsymbol{V}_{h}^{n+1}-d_{t} \boldsymbol{V}_{f, h}^{n+1}\right) \cdot d_{t} \boldsymbol{V}_{h}^{n+1} d \boldsymbol{x} \\
=- & \frac{\rho_{q} \phi \Delta t^{2}}{2}\left\|d_{t} \boldsymbol{V}_{h}^{n+1}\right\|_{L^{2}\left(\Omega^{p}\right)}^{2}+\frac{\rho_{q} \phi \Delta t^{2}}{2}\left\|d_{t} \boldsymbol{V}_{f, h}^{n+1}\right\|_{L^{2}\left(\Omega^{p}\right)}^{2}-\frac{\rho_{q} \phi \Delta t^{2}}{2}\left\|d_{t}\left(\boldsymbol{V}_{f, h}^{n+1}-\boldsymbol{V}_{h}^{n+1}\right)\right\|_{L^{2}\left(\Omega^{p}\right)}^{2} \\
= & -\frac{\rho_{q} \phi \Delta t^{2}}{2}\left\|d_{t} \boldsymbol{V}_{h}^{n+1}\right\|_{L^{2}\left(\Omega^{p}\right)}^{2}+\frac{\rho_{q} \phi \Delta t^{2}}{2}\left\|d_{t} \boldsymbol{V}_{f, h}^{n+1}\right\|_{L^{2}\left(\Omega^{p}\right)}^{2}-\frac{m \Delta t^{2}}{2}\left\|d_{t} \boldsymbol{q}_{h}^{n+1}\right\|_{L^{2}\left(\Omega^{p}\right)}^{2} .
\end{aligned}
$$

From (4.9) and (4.10), and using the discrete energy defined by (4.4)-(4.6), we have

$$
\begin{gathered}
\mathcal{E}^{n+1}+\frac{\rho_{f} \Delta t^{2}}{2}\left\|d_{t} \boldsymbol{v}_{h}^{n+1}\right\|_{L^{2}\left(\Omega^{f}\right)}^{2}+2 \mu_{f} \Delta t\left\|D\left(\boldsymbol{v}_{h}^{n+1}\right)\right\|_{L^{2}\left(\Omega^{f}\right)}^{2}+\frac{\rho_{m} h}{2}\left\|\boldsymbol{v}_{h}^{n+1}-\boldsymbol{\xi}_{h}^{n}\right\|_{L^{2}\left(\Gamma^{f s}\right)}^{2} \\
+\frac{(1-\phi) \rho_{s} \Delta t^{2}}{2}\left\|d_{t} \boldsymbol{V}_{h}^{n+1}\right\|_{L^{2}\left(\Omega^{p}\right)}^{2}+\frac{\phi \rho_{q} \Delta t^{2}}{2}\left\|d_{t} \boldsymbol{V}_{f, h}^{n+1}\right\|_{L^{2}\left(\Omega^{p}\right)}^{2}+\frac{\Delta t^{2}}{2}\left\|d_{t} \boldsymbol{U}_{h}^{n+1}\right\|_{E}^{2} \\
+\Delta t\left\|\boldsymbol{\kappa}^{-1} \boldsymbol{q}_{h}^{n+1}\right\|_{L^{2}\left(\Omega^{p}\right)}^{2}+\frac{\Delta t^{2}}{2 M}\left\|d_{t} p_{p, h}^{n+1}\right\|_{L^{2}\left(\Omega^{p}\right)}^{2}+\frac{\rho_{m} h}{2}\left\|\boldsymbol{V}_{h}^{n+1}-\boldsymbol{\xi}_{h}^{n}\right\|_{L^{2}\left(\Gamma^{p s}\right)}^{2}
\end{gathered}
$$




$$
\begin{gathered}
+\frac{\rho_{m} h}{2}\left\|\boldsymbol{\xi}_{h}^{n+1}-\boldsymbol{V}_{h}^{n+1}\right\|_{L^{2}\left(\Gamma^{p s}\right)}^{2}+\frac{\rho_{m} h}{2}\left\|\boldsymbol{\xi}_{h}^{n+1}-\boldsymbol{v}_{h}^{n+1}\right\|_{L^{2}\left(\Gamma^{f s}\right)}^{2}+\frac{\Delta t \alpha_{f}}{2}\left\|\left(\boldsymbol{v}_{h}^{n+1}-\boldsymbol{v}_{h}^{n}\right) \cdot \boldsymbol{n}^{f}\right\|_{L^{2}\left(\Gamma^{f p}\right)}^{2} \\
+\frac{\Delta t \alpha_{s}}{2}\left\|\left(\boldsymbol{V}_{h}^{n+1}-\boldsymbol{V}_{h}^{n}\right) \cdot \boldsymbol{\tau}^{f}\right\|_{L^{2}\left(\Gamma^{f p}\right)}^{2}+\frac{\Delta t \alpha_{p}}{2}\left\|\left(p_{p, h}^{n+1}-p_{p, h}^{n}\right)\right\|_{L^{2}\left(\Gamma^{f p}\right)}^{2}=\mathcal{E}^{n} \\
-\Delta t c_{s}\left(\boldsymbol{v}_{h}^{n+1}-\boldsymbol{V}_{h}^{n}, \boldsymbol{v}_{h}^{n+1}\right)+\Delta t c_{s}\left(\boldsymbol{v}_{h}^{n+1}-\boldsymbol{V}_{h}^{n+1}, \boldsymbol{V}_{h}^{n+1}\right)+\Delta t c_{p}\left(p_{p, h}^{n+1}-p_{p, h}^{n}, \boldsymbol{v}_{h}^{n+1}\right) \\
-\Delta t \int_{\Gamma_{\text {in }}^{f}} p_{i n}\left(t^{n+1}\right) \boldsymbol{v}_{h}^{n+1} \cdot \boldsymbol{n}^{f} d x-\Delta t \int_{\Gamma_{\text {out }}^{f}} p_{\text {out }}\left(t^{n+1}\right) \boldsymbol{v}_{h}^{n+1} \cdot \boldsymbol{n}^{f} d x
\end{gathered}
$$

Note that we can rewrite terms $-\Delta t c_{s}\left(\boldsymbol{v}_{h}^{n+1}-\boldsymbol{V}_{h}^{n}, \boldsymbol{v}_{h}^{n+1}\right)+\Delta t c_{s}\left(\boldsymbol{v}_{h}^{n+1}-\boldsymbol{V}_{h}^{n+1}, \boldsymbol{V}_{h}^{n+1}\right)$ as

$$
-\Delta t c_{s}\left(\boldsymbol{v}_{h}^{n+1}-\boldsymbol{V}_{h}^{n+1}, \boldsymbol{v}_{h}^{n+1}-\boldsymbol{V}_{h}^{n+1}\right)-\Delta t c_{s}\left(\boldsymbol{V}_{h}^{n+1}-\boldsymbol{V}_{h}^{n}, \boldsymbol{v}_{h}^{n+1}\right) .
$$

The first term in (4.12) is equal to $-\gamma^{f p} \Delta t\left\|P_{\tau}^{f}\left(\left.\boldsymbol{v}_{h}^{n+1}\right|_{\Gamma^{f p}\left(t^{n}\right)}-\boldsymbol{V}_{h}^{n+1}\right)\right\|_{L^{2}\left(\Gamma^{f p}\right)}^{2}$ and can be combined with the dissipative terms on the left hand side. To bound the second term in (4.12), we use the Young's inequality with $\epsilon_{1}>0$, trace inequality with constant $C_{T}$, Poincaré inequality with constant $C_{P}$, and Korn's inequality with constant $C_{K}[13]$ as follows

$$
\begin{gathered}
\left|\Delta t c_{s}\left(\boldsymbol{V}_{h}^{n+1}-\boldsymbol{V}_{h}^{n}, \boldsymbol{v}_{h}^{n+1}\right)\right| \leq \frac{\Delta t \gamma^{f p} \epsilon_{1}}{2}\left\|\boldsymbol{V}_{h}^{n+1}-\boldsymbol{V}_{h}^{n}\right\|_{L^{2}\left(\Gamma^{f p}\right)}^{2}+\frac{\Delta t \gamma^{f p}}{2 \epsilon_{1}}\left\|\boldsymbol{v}_{h}^{n+1}\right\|_{L^{2}\left(\Gamma^{f p}\right)}^{2} \\
\leq \frac{\Delta t \gamma^{f p} \epsilon_{1}}{2}\left\|\boldsymbol{V}_{h}^{n+1}-\boldsymbol{V}_{h}^{n}\right\|_{L^{2}\left(\Gamma^{f p}\right)}^{2}+\frac{\Delta t \gamma^{f p} C_{T}^{2} C_{K}^{2} C_{P}}{2 \epsilon_{1}}\left\|\boldsymbol{D}\left(\boldsymbol{v}_{h}^{n+1}\right)\right\|_{L^{2}\left(\Omega^{p}\right)}^{2} .
\end{gathered}
$$

Term $\Delta t c_{p}\left(p_{p, h}^{n+1}-p_{p, h}^{n}, \boldsymbol{v}_{h}^{n+1}\right)$ is bounded in a similar way using Young's inequality with $\epsilon_{2}>0$, trace inequality, Poincaré inequality and Korn's inequality as follows

$$
\begin{gathered}
\left|\Delta t c_{p}\left(p_{p, h}^{n+1}-p_{p, h}^{n}, \boldsymbol{v}_{h}^{n+1}\right)\right| \leq \frac{\epsilon_{2} \Delta t}{2}\left\|p_{p, h}^{n+1}-p_{p, h}^{n}\right\|_{L^{2}\left(\Gamma^{f p}\right)}^{2}+\frac{\Delta t}{2 \epsilon_{2}}\left\|\boldsymbol{v}_{h}^{n+1}\right\|_{L^{2}\left(\Gamma^{f p}\right)}^{2} \\
\leq \frac{\epsilon_{2} \Delta t}{2}\left\|p_{p, h}^{n+1}-p_{p, h}^{n}\right\|_{L^{2}\left(\Gamma^{f p}\right)}^{2}+\frac{\Delta t C_{T}^{2} C_{K}^{2} C_{P}}{2 \epsilon_{2}}\left\|\boldsymbol{D}\left(\boldsymbol{v}_{h}^{n+1}\right)\right\|_{L^{2}\left(\Omega^{f}\right)}^{2} .
\end{gathered}
$$

Finally, we bound the terms due to the fluid boundary conditions using the Young's inequality with $\epsilon_{3}=\frac{2 C_{T}^{2} C_{K}^{2} C_{P}}{\mu_{f}}$, trace inequality, Poincaré inequality and Korn's inequality as follows

$$
\begin{gathered}
-\Delta t \int_{\Gamma_{\text {in }}^{f}} p_{\text {in }}\left(t^{n+1}\right) \boldsymbol{v}_{h}^{n+1} \cdot \boldsymbol{n}^{f} d x-\Delta t \int_{\Gamma_{\text {out }}^{f}} p_{\text {out }}\left(t^{n+1}\right) \boldsymbol{v}_{h}^{n+1} \cdot \boldsymbol{n}^{f} d x \\
\leq \frac{\Delta t C_{T}^{2} C_{K}^{2} C_{P}}{\mu_{f}}\left(\left\|p_{\text {in }}\left(t^{n+1}\right)\right\|_{L^{2}\left(\Gamma_{\text {in }}^{f}\right)}^{2}+\left\|p_{\text {out }}\left(t^{n+1}\right)\right\|_{L^{2}\left(\Gamma_{\text {out }}^{f}\right)}^{2}\right)+\frac{\Delta t \mu_{f}}{2}\left\|\boldsymbol{D}\left(\boldsymbol{v}_{h}^{n+1}\right)\right\|_{L^{2}\left(\Omega^{f}\right)^{2}}^{2} .
\end{gathered}
$$

Using the triangle inequality, setting $\epsilon_{1}=\frac{2 \gamma^{f p} C_{T}^{2} C_{K}^{2} C_{P}}{\mu_{f}}$ and $\epsilon_{2}=\frac{2 C_{T}^{2} C_{K}^{2} C_{P}}{\mu_{f}}$ and combining terms in (4.13), (4.14) and (4.15) with the equivalent terms on the left hand side, we prove the desired estimate.

Remark 4. Stability conditions in (4.7) are independent of the space and time discretization parameters. Hence, we do not have a limitation on the choice of the time step. Furthermore, the stability conditions do not depend on the fluid and structure densities, and therefore the scheme is not affected by the instabilities related to the added mass effect. 
Remark 5. Note that there is no requirement on $\alpha_{f}$ needed in order to achieve stability, and hence one can choose $\alpha_{f}=0$. However, we consider other values for $\alpha_{f}$ since our numerical results indicate that larger values of $\alpha_{f}$ can help to improve the accuracy of the approximation of the fluid velocity at the fluid-poroelastic medium interface. Since the combination parameters introduced here have a similar role as the combination parameters introduced in [8], we propose to use

$$
\alpha_{f}=\frac{L}{\pi} \tanh \left(\frac{\pi H}{L}\right) \frac{\rho_{b}+a \Delta t^{2}}{\Delta t+\kappa\left(\rho_{b}-\rho_{q}+a \Delta t^{2}\right)}
$$

where $H$ is the average thickness of $\Omega^{p}, a=\frac{1}{R^{2}}\left(\frac{2 \mu_{s} \lambda_{s}}{\lambda_{s}+2 \mu_{s}}+2 \mu_{s}\right)$, and $\kappa$ is the minimum eigenvalue of the permeability tensor.

Furthermore, assuming the numerical scheme remains stable, following the approach in [8], we propose to use

$$
\alpha_{p}=\frac{\Delta t}{\rho_{f} \mu_{\max }^{\mathcal{M}}}, \quad \alpha_{s}=\alpha_{p}
$$

where $\mu_{\text {max }}^{\mathcal{M}}$ is the largest eigenvalue of the added-mass operator, derived in [20] for an idealized rectangular fluid domain of height $R$ and length $L$, given by

$$
\mu_{\max }^{\mathcal{M}}=\frac{L}{\pi \tanh \left(\frac{\pi R}{L}\right)} .
$$

This choice of combination parameters is obtained by analyzing the stability of a simplified problem, which is often done when designing partitioned algorithms for fluid-structure interaction problems $[8,5,6,16]$.

\section{Numerical results}

In order to verify the accuracy and stability of the proposed numerical scheme (3.4)-(3.7), test the convergence and investigate the properties of the mathematical model, we present two numerical examples. In Example 1 we compare our results to the ones obtained by a strongly coupled scheme with the assumption of the rigid vessel walls. Then, we investigate the order of the time and space convergence for the full, moving wall problem. We focus on a classical benchmark problem $[33,11,5,9,14]$ used to validate numerical methods for FSI problems in hemodynamics.

In Example 2 we investigate the effects of the material properties of the poroelastic region on the fluid flow in a cylinder. We also compare our results to the ones obtained by considering the fluid-structure interaction model only.

\subsection{Example 1}

In this example we consider the classical benchmark problem used to test FSI algorithms for blood flow $[33,11,5,9,14]$. This problem was implemented in two spatial dimensions in 
Fortran 90. The thin structure model used in this test case is a linearly elastic Koiter shell model

$$
\begin{array}{ll}
\rho_{m} h \frac{\partial^{2} \eta_{z}}{\partial t^{2}}-C_{2} \frac{\partial \eta_{r}}{\partial z}-C_{1} \frac{\partial^{2} \eta_{z}}{\partial z^{2}}=f_{z} & \text { on } \Gamma^{s} \times(0, T), \\
\rho_{m} h \frac{\partial^{2} \eta_{r}}{\partial t^{2}}+C_{0} \eta_{r}+C_{2} \frac{\partial \eta_{z}}{\partial z}-C_{3} \frac{\partial^{2} \eta_{r}}{\partial z^{2}}=f_{r} & \text { on } \Gamma^{s} \times(0, T),
\end{array}
$$

where $\eta_{z}$ denotes the longitudinal displacement, $\eta_{r}$ denotes the radial displacement, and

$$
\begin{aligned}
C_{0} & =\frac{h}{R^{2}}\left(\frac{2 \mu_{m} \lambda_{m}}{\lambda_{m}+2 \mu_{m}}+2 \mu_{m}\right)\left(1+\frac{h^{2}}{12 R^{2}}\right), & C_{1} & =h\left(\frac{2 \mu_{m} \lambda_{m}}{\lambda_{m}+2 \mu_{m}}+2 \mu_{m}\right), \\
C_{2} & =\frac{h}{R} \frac{2 \mu_{m} \lambda_{m}}{\lambda_{m}+2 \mu_{m}}, & C_{3} & =\frac{h^{3}}{6 R^{2}} \frac{2 \mu_{m} \lambda_{m}}{\lambda_{m}+2 \mu_{m}} .
\end{aligned}
$$

The coefficients $\mu_{m}$ and $\lambda_{m}$ are the Lamé coefficients for the shell.

The values of the parameters used in this example are given in Table 1 . The reference

\begin{tabular}{|c|c|c|c|}
\hline Parameters & Values & Parameters & Values \\
\hline \multicolumn{4}{|l|}{ Geometry: } \\
\hline $\operatorname{Radius} R(\mathrm{~cm})$ & 0.5 & Length $L(\mathrm{~cm})$ & 5 \\
\hline \multicolumn{4}{|l|}{ Fluid: } \\
\hline Fluid density $\rho_{f}\left(\mathrm{~g} / \mathrm{cm}^{3}\right)$ & 1 & Fluid viscosity $\mu_{f}(\mathrm{~g} / \mathrm{cm} \mathrm{s})$ & 0.035 \\
\hline \multicolumn{4}{|l|}{ Thin structure (shell): } \\
\hline Shell thickness $h(\mathrm{~cm})$ & 0.1 & Shell density $\rho_{m}\left(\mathrm{~g} / \mathrm{cm}^{3}\right)$ & 1.1 \\
\hline Lamé coeff. $\mu_{m}\left(\right.$ dyne $\left./ \mathrm{cm}^{2}\right)$ & $3.574 \times 10^{5}$ & Lamé coeff. $\lambda_{m}\left(\right.$ dyne $\left./ \mathrm{cm}^{2}\right)$ & $1.428 \times 10^{6}$ \\
\hline \multicolumn{4}{|l|}{ Poroelasticity: } \\
\hline Porosity $\phi$ & 0.9 & Slip rate $\gamma^{f p}\left(\mathrm{~cm}^{3} \mathrm{~s} / \mathrm{g}\right)$ & $10^{2}$ \\
\hline Fluid density $\rho_{q}\left(\mathrm{~g} / \mathrm{cm}^{3}\right)$ & 1.027 & Structure density $\rho_{s}\left(\mathrm{~g} / \mathrm{cm}^{3}\right)$ & 1.3 \\
\hline Lamé coeff. $\mu_{s}\left(\right.$ dyne $\left./ \mathrm{cm}^{2}\right)$ & $2.227 \times 10^{5}$ & Lamé coeff. $\lambda_{s}\left(\right.$ dyne $\left./ \mathrm{cm}^{2}\right)$ & $1.098 \times 10^{6}$ \\
\hline Hydraulic conductivity $\kappa\left(\mathrm{cm}^{3} \mathrm{~s} / \mathrm{g}\right)$ & $9.6154 \times 10^{-6} \boldsymbol{I}$ & Biot-Willis constant $\alpha$ & 1 \\
\hline Biot modulus $M\left(\right.$ dyne $\left./ \mathrm{cm}^{2}\right)$ & $10^{4}$ & & \\
\hline
\end{tabular}
domain $\Omega=\Omega^{f} \cup \Omega^{p}$ is defined as a rectangle $[0, L] \times[-R, R]$. The interface $\Gamma^{f p}$ between the fluid and the poroelastic material is defined as

$$
x=-0.8 \cos (t)+2.5, \quad y=0.4 \sin (t)+0.5 \quad \text { for } t \in(\pi, 2 \pi) .
$$

Table 1: Geometry, fluid, structure, and poroelasticity parameters used in Example 1.

\subsubsection{Comparison with a strongly coupled scheme}

In this subsection we compare our results to the ones obtained using a strongly coupled scheme. A design of a strongly coupled or a monolithic solver for the fully coupled problem (2.38) is non trivial because the appropriate convergence parameters and/or preconditioners need to be determined, which is out of scope of this paper. Since the kinematically coupled $\beta$ scheme that we use to decouple fluid and the thin structure was previously compared to a solution obtained by a monolithic solver in [11], we focus on the decoupling between 
the fluid and a poroelastic medium. Hence, we assume that the wall $\Gamma^{s}$ is rigid, and compare our results to the ones obtained by the Robin-Robin scheme for the interaction between a fluid and a poroelastic material presented in [8].

At the inlet fluid boundary $\Gamma_{i n}^{f}$ we impose a steady, parabolic velocity profile with the maximum amplitude of $5 \mathrm{~cm} / \mathrm{s}$, and assume zero radial velocity. At the outlet fluid boundary we impose $\boldsymbol{\sigma}^{f} \boldsymbol{n}_{\text {out }}^{f}=0$. On $\Gamma^{f s}$ we impose the no-slip condition and on $\Gamma^{p s}$ we assume zero displacement. The simulations are preformed until the steady state is reached. The reference solution is generated using the Robin-Robin algorithm presented in [8] with high grid resolution $\left(\Delta t=10^{-4}\right.$ and $\left.\Delta x=2 \cdot 10^{-2}\right)$. In order to show the convergence of our results to the reference solution, we refine at the same rate both in time and space:

$$
(\Delta t, \Delta x) \in\left\{\left(\frac{0.1}{2^{i}}, \frac{0.2}{2^{i}}\right)\right\}_{i=0}^{2} .
$$

We use the same combination parameters for both schemes, $\alpha_{f}=0.13 \Delta t^{-1}+3.05 \cdot 10^{5} \Delta t, \alpha_{p}=$ $10 \Delta t, \alpha_{s}=\alpha_{p}$. Figure 2 shows the relative error between the fluid velocity $\left(e_{v}\right)$, Darcy velocity $\left(e_{q}\right)$ and displacement $\left(e_{U}\right)$ obtained using our scheme and the reference solution. The error was computed at the end of simulation time, when the steady state solution was reached. We observe the error decreasing with the first-order rate as we decrease the time step and mesh size. Figure 3 shows a comparison of the fluid velocity, Darcy velocity and displacement obtained with the loosely coupled scheme when $i=2$ (bottom) and the strongly coupled scheme (top). An excellent agreement is observed.
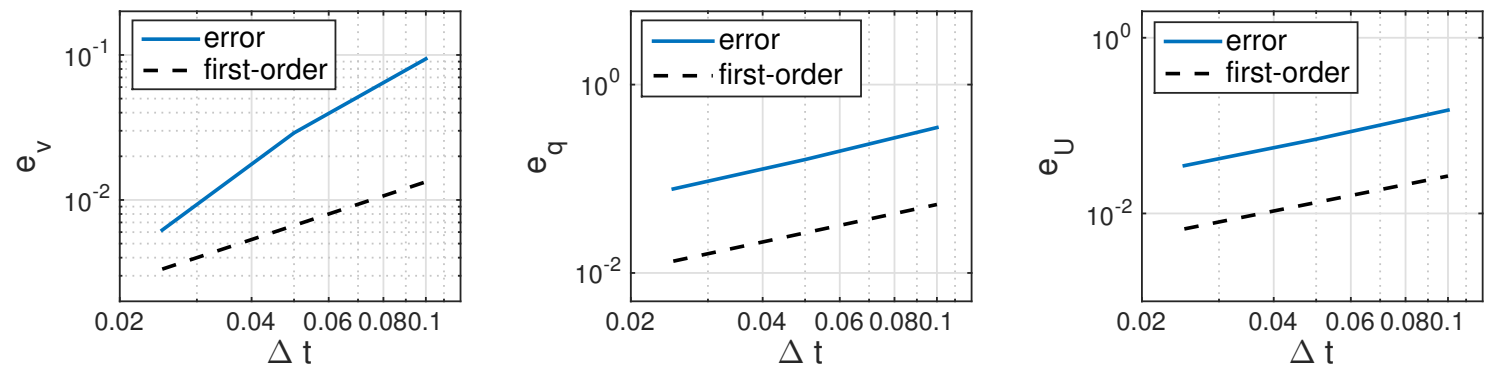

Figure 2: Relative error between the solutions obtained using our scheme and a reference solution generated by the strongly coupled Robin-Robin algorithm from [8]. Left: Fluid velocity. Middle: Darcy velocity. Right: Poroelastic material displacement.

Finally, we note that it was sufficient to use $\alpha_{p}=10 \Delta t$ and $\alpha_{s}=\alpha_{p}$ in order to achieve stability. This is less restrictive than the prediction (4.7) from the stability analysis, indicating that the scheme enjoys better stability properties than prescribed by (4.7).

\subsubsection{Time and space convergence}

In this example we study the convergence of scheme (3.4)-(3.7) in time and space. The time discretization of each sub-problem is performed using a first-order accurate method. We expect the discretization of the coupling conditions between the fluid and the poroelastic structure to have the accuracy of $\mathcal{O}(\Delta t)$ [18]. Furthermore, it was shown in [28] that the 

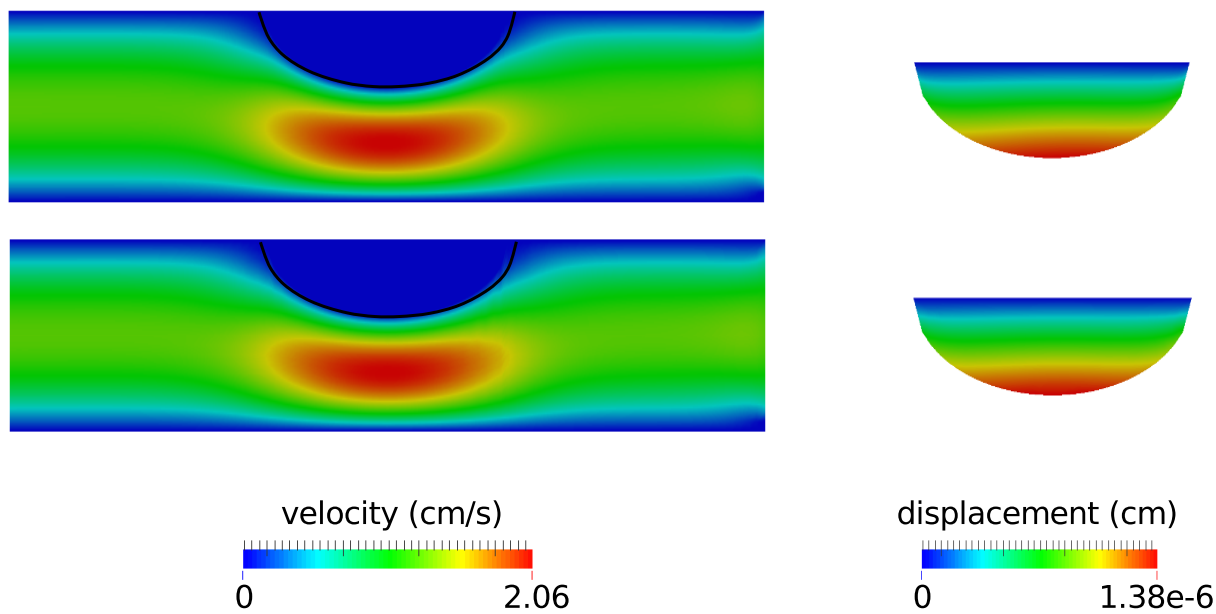

Figure 3: Comparison of the fluid and Darcy velocity (left) and poroelastic material displacement (right) obtained by a strongly coupled scheme (top) by Badia et al [8] and the loosely coupled scheme (3.4)-(3.7) (bottom). The results were obtained under the assumption of rigid lateral walls.

kinematically coupled $\beta$ scheme features a sub-optimal $\mathcal{O}\left(\Delta t^{1 / 2}\right)$ convergence when $\beta=0$. However, a better rate of convergence was observed numerically when $\beta=1$ in [11]. Hence we expect to observe a sub-optimal convergence of scheme (3.4)-(3.7) when $\beta=0$ and optimal convergence when $\beta=1$.

We use parameters listed in Table 1 , and values of combination parameters $\alpha_{s}=10^{-3}, \alpha_{p}=$ $10^{-3}$ and $\alpha_{f}=10^{4}$. The flow is driven by the time-dependent pressure data

$$
p_{\text {in }}(t)=\left\{\begin{array}{ll}
\frac{p_{\max }}{2}\left(1-\cos \left(\frac{2 \pi t}{t_{\text {max }}}\right)\right) & \text { it } t \leq t_{\text {max }}, \\
0 & \text { it } t>t_{\text {max }},
\end{array} \quad p_{\text {out }}(t)=0, \forall t \in(0, T),\right.
$$

where $p_{\max }=2 \cdot 10^{4}$ dyne $/ \mathrm{cm}^{2}$ and $t_{\max }=5 \mathrm{~ms}$. The problem was solved over the time interval $[0,14] \mathrm{ms}$.

Figure 4 shows the propagation of the fluid pressure wave and the velocity streamlines at times $t=5,8,11$ and $14 \mathrm{~ms}$. As the pressure wave reaches the occluded poroelastic region at $t=8 \mathrm{~ms}$, we observe the increase of the Darcy pressure in the poroelastic material. As the pressure wave continues to propagate through the channel, we observe the creation of several recirculation zones.

The displacement of the elastic structure and the poroelastic material is shown in Figure 5 at times $t=8 \mathrm{~ms}$ and $t=14 \mathrm{~ms}$. The left panel shows the radial displacement of the poroelastic region superimposed with the reference configuration. The right panel shows the magnitude of the displacement superimposed with the displacement vectors indicating the direction of deformation. We observe that the poroelastic region is first displaced in the radial direction and then moved longitudinally as the peak pressure wave reaches the end of the occluded section of the fluid domain at $t=14 \mathrm{~ms}$.

The results in Figures 4 and 5 are obtained with the time step $\Delta t=10^{-4}$ and the characteristic mesh size $\Delta x=0.04$ in both fluid and poroelastic region. The number of nodes in the fluid mesh is 7913 , and the number of triangles is 15360 . In the poroelastic 

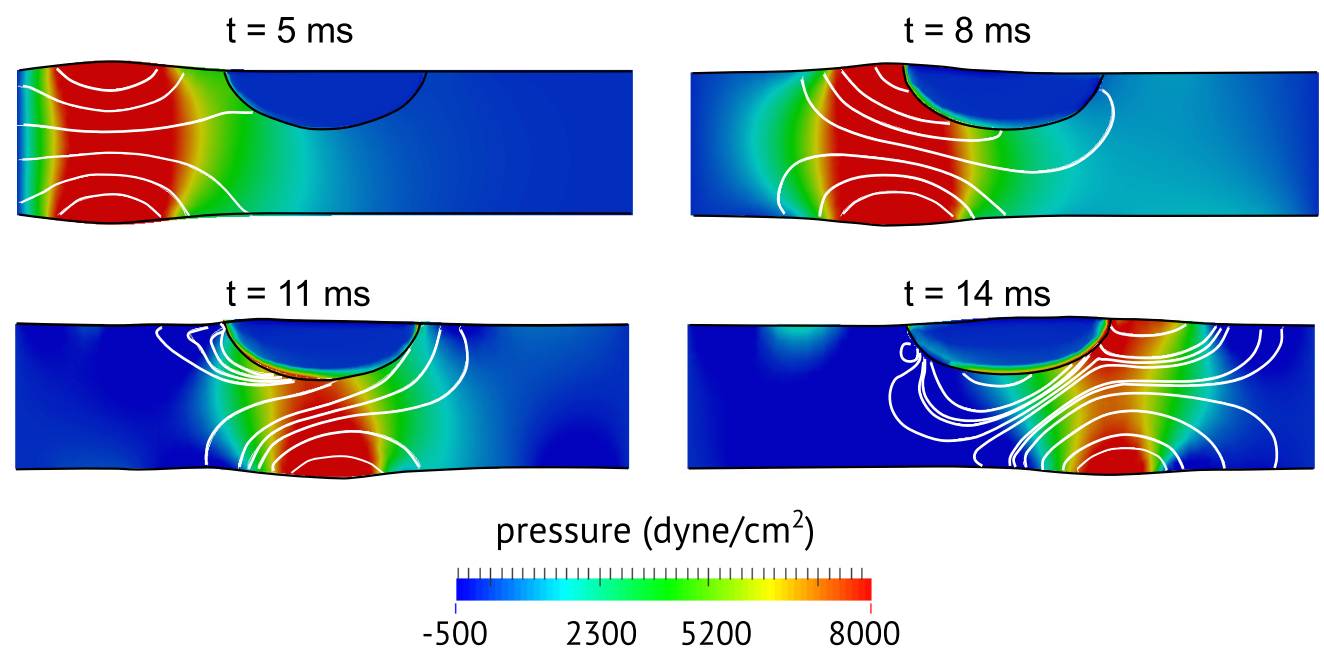

Figure 4: Propagation of the fluid pressure wave, velocity streamlines and Darcy pressure in the poroelastic material at four different times. As the fluid reaches the poroelastic region, the Darcy pressure increases. Several recirculation zones form as the pressure wave continues to propagate through the channel.

region, we have 846 nodes and 1562 triangles. We use the value of $\beta=1$. To approximate the fluid velocity and pressure we use P1-iso-P2 and P1 elements. We use P1 elements to approximate the structure displacement, as well as the Darcy pressure and velocity.

In order to test the convergence properties, we study the time and space convergence using values $\beta=1$ and $\beta=0$. To study convergence in time, we define the reference solution to be the one obtained with $\Delta t=10^{-6}$, and the relative errors between the reference solution and the computed solution in the fluid velocity, Darcy velocity, poroelastic material displacement and the thin structure displacement as

$$
e_{v}=\frac{\left\|\boldsymbol{v}-\boldsymbol{v}_{r e f}\right\|_{L^{2}\left(\Omega^{f}\right)}}{\left\|\boldsymbol{v}_{r e f}\right\|_{L^{2}\left(\Omega^{f}\right)}}, e_{q}=\frac{\left\|\boldsymbol{q}-\boldsymbol{q}_{r e f}\right\|_{L^{2}\left(\Omega^{p}\right)}}{\left\|\boldsymbol{q}_{r e f}\right\|_{L^{2}\left(\Omega^{p}\right)}}, e_{U}=\frac{\left\|\boldsymbol{U}-\boldsymbol{U}_{r e f}\right\|_{L^{2}\left(\Omega^{p}\right)}}{\left\|\boldsymbol{U}_{r e f}\right\|_{L^{2}\left(\Omega^{p}\right)}}, e_{\eta}=\frac{\left\|\boldsymbol{\eta}-\boldsymbol{\eta}_{r e f}\right\|_{L^{2}\left(\Gamma^{s}\right)}}{\left\|\boldsymbol{\eta}_{r e f}\right\|_{L^{2}\left(\Gamma^{s}\right)}} .
$$

The relative errors are measured at $t=10 \mathrm{~ms}$ for both $\beta=1$ and $\beta=0$. Figure 6 shows the comparison of the relative errors obtained in both cases. We observe that the relative errors obtained with $\beta=1$ are smaller than the ones obtained with $\beta=0$ for all the measured variables. While in the case when $\beta=1$ we observe the first-order convergence in time, a smaller rate is observed when $\beta=0$. This is consistent with the convergence properties of the kinematically coupled $\beta$-scheme for fluid-structure interaction reported in $[28,11]$. Detailed report of the relative errors obtained in this example is given in Table 2.

To study convergence in space, we define the reference solution to be the one obtained with $\Delta x=\Delta x_{f}=\Delta x_{p}=0.02$ and we set $\Delta t=10^{-5}$. The relative errors at $t=10 \mathrm{~ms}$ for both $\beta=1$ and $\beta=0$ are shown in Table 3. There are no significant differences between the relative errors obtained with $\beta=1$ and with $\beta=0$. Both cases indicate the first order convergence in space.

\subsection{Example 2}

In this example we extend the implementation of the benchmark problem considered in Example 1 to three spatial dimensions. This problem was implemented in FreeFem $++[40]$. 


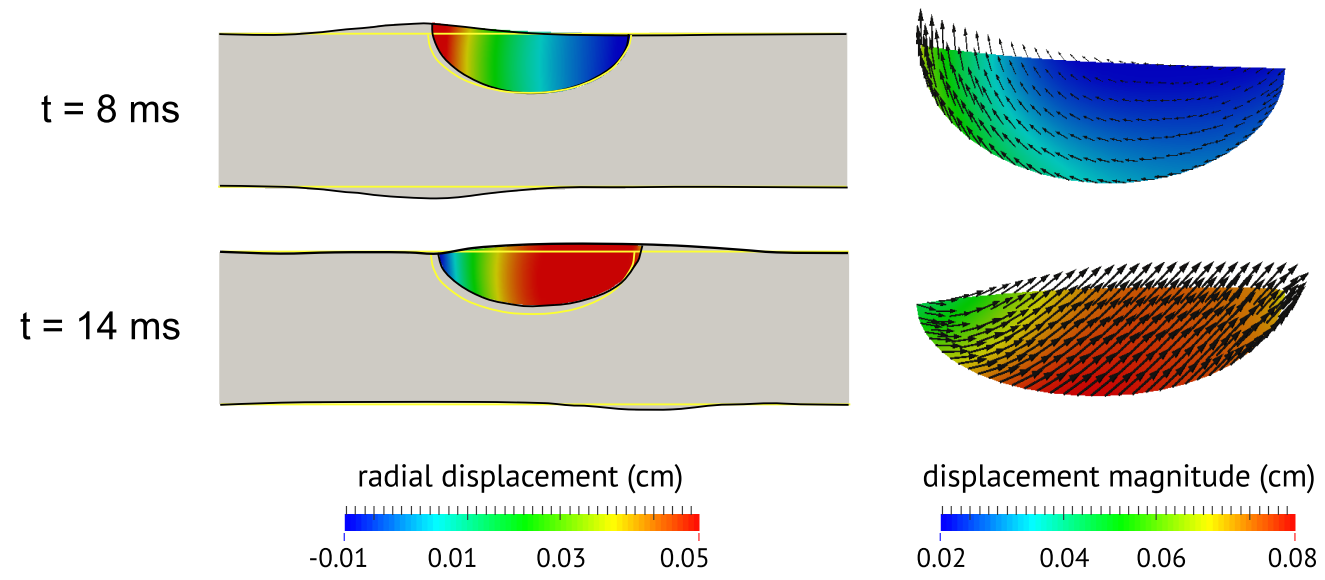

Figure 5: Displacement of the elastic wall and the poroelastic material at $t=8 \mathrm{~ms}$ and $t=14 \mathrm{~ms}$. The left panel shows the radial displacement superimposed with the reference configuration. The right panel shows the magnitude of the displacement superimposed with the deformation direction vectors.

\begin{tabular}{|c|c|c|c|c|c|c|c|c|c|}
\hline \multirow{6}{*}{$\beta=1:$} & $\triangle t$ & $e_{v}$ & rate & $e_{q}$ & rate & $e_{U}$ & rate & $e_{\eta}$ & rate \\
\hline & $2 \cdot 2 \cdot 10^{-4}$ & $6.6 \mathrm{e}-2$ & - & $6.5 \mathrm{e}-2$ & - & $1.6 \mathrm{e}-1$ & - & $\overline{-1.2 \mathrm{e}-1}$ & - \\
\hline & $10^{-4}$ & $2.9 \mathrm{e}-2$ & 1.2 & $3.0 \mathrm{e}-2$ & 1.1 & $8.3 e-2$ & 1.0 & $7.3 e-2$ & 0.8 \\
\hline & $5 \cdot 10^{-5}$ & $1.6 \mathrm{e}-2$ & 0.9 & $1.8 \mathrm{e}-2$ & 0.7 & $4.4 \mathrm{e}-2$ & 0.9 & $3.9 \mathrm{e}-2$ & 0.9 \\
\hline & $10^{-5}$ & $3.4 \mathrm{e}-3$ & 1.0 & $5.0 \mathrm{e}-3$ & 0.8 & $9.7 \mathrm{e}-3$ & 0.9 & $7.7 \mathrm{e}-3$ & 1.0 \\
\hline & $5 \times 10^{-6}$ & $1.5 \mathrm{e}-3$ & 1.1 & $2.4 \mathrm{e}-3$ & 1.1 & $4.4 \mathrm{e}-3$ & 1.1 & $3.5 \mathrm{e}-3$ & 1.2 \\
\hline \multirow{6}{*}{$\beta=0:$} & $\triangle t$ & $e_{v}$ & rate & $e_{q}$ & rate & $e_{U}$ & rate & $e_{\eta}$ & rate \\
\hline & $2 \cdot 10^{-4}$ & $5.1 \mathrm{e}-1$ & - & $3.5 \mathrm{e}-1$ & - & $3.1 \mathrm{e}-1$ & - & $\overline{c 1.1 \mathrm{e}+0}$ & - \\
\hline & $10^{-4}$ & $2.7 \mathrm{e}-1$ & 0.9 & $2.1 \mathrm{e}-1$ & 0.8 & $2.0 \mathrm{e}-1$ & 0.6 & $1.9 \mathrm{e}-1$ & 2.6 \\
\hline & $5 \cdot 10^{-5}$ & $1.4 \mathrm{e}-1$ & 0.9 & $1.2 \mathrm{e}-1$ & 0.9 & $1.2 \mathrm{e}-1$ & 0.8 & $1.0 \mathrm{e}-1$ & 0.9 \\
\hline & $10^{-5}$ & $2.7 \mathrm{e}-2$ & 1.0 & $2.4 \mathrm{e}-2$ & 1.0 & $2.5 \mathrm{e}-2$ & 0.9 & $2.1 \mathrm{e}-2$ & 1.0 \\
\hline & $5 \times 10^{-6}$ & $1.2 \mathrm{e}-2$ & 1.2 & $1.1 \mathrm{e}-2$ & 1.1 & $1.1 \mathrm{e}-2$ & 1.1 & $9.3 \mathrm{e}-3$ & 1.1 \\
\hline
\end{tabular}

Table 2: Convergence in time. Top: $\beta=1$. Bottom: $\beta=0$.

The thin structure model used in this test case is a linearly elastic membrane model proposed in $[31,30,23]$, given in the weak form as

$$
\rho_{m} h \int_{\Gamma^{s}} \frac{\partial^{2} \boldsymbol{\eta}}{\partial t^{2}} \cdot \boldsymbol{\zeta} d \Gamma+h \int_{\Gamma^{s}} \boldsymbol{\Pi}_{\gamma}(\boldsymbol{\eta}): \nabla_{\gamma} \boldsymbol{\zeta} d \Gamma=\int_{\Gamma^{s}} \boldsymbol{f} \cdot \boldsymbol{\zeta} d \Gamma
$$

where $\boldsymbol{\eta}=\left(\eta_{x}, \eta_{y}, \eta_{z}\right)$ denotes the structure displacement. For a linearly elastic, isotropic structure

$$
\boldsymbol{\Pi}_{\gamma}(\boldsymbol{\eta})=\frac{E}{1+\sigma^{2}} \frac{\nabla_{\gamma} \boldsymbol{\eta}+\nabla_{\gamma}^{T} \boldsymbol{\eta}}{2}+\frac{E \sigma}{1-\sigma^{2}} \nabla_{\gamma} \cdot \boldsymbol{\eta}
$$



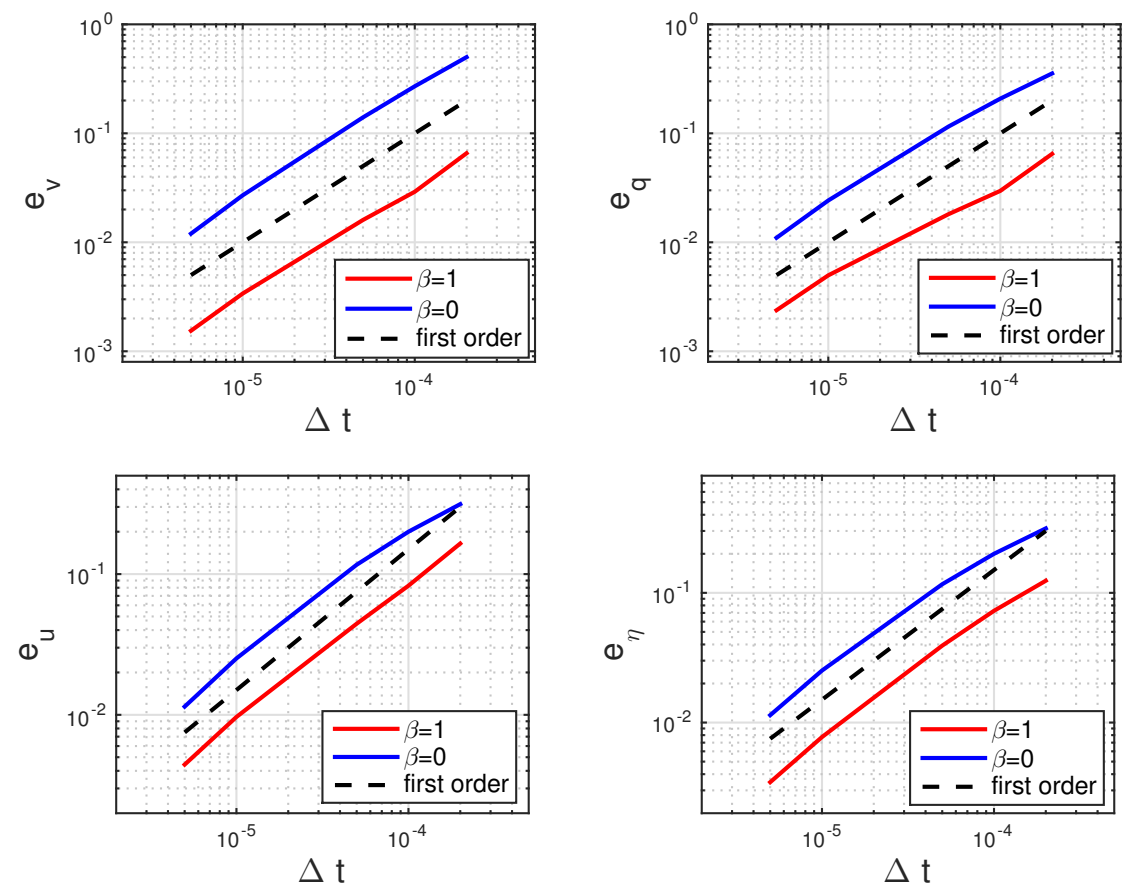

Figure 6: Convergence in time obtained with $\beta=1$ and $\beta=0$. Top left: Fluid velocity. Top right: Darcy velocity. Bottom left: Poroelastic material displacement. Bottom right: Thin structure displacement. Smaller relative error in all cases is obtained with $\beta=1$. Numerical results indicate first order convergence in time when $\beta=1$.

\begin{tabular}{|c|c|c|c|c|c|c|c|c|c|}
\hline \multirow{5}{*}{$\beta=1:$} & $\triangle x$ & $e_{v}$ & rate & $e_{q}$ & rate & $e_{U}$ & rate & $e_{\eta}$ & rate \\
\hline & "0.34 & $3.8 \mathrm{e}-1$ & - & $6.1 \mathrm{e}-1$ & - & $3.0 \mathrm{e}-1$ & - & $2.7 \mathrm{e}-1$ & - \\
\hline & 0.17 & $2.0 \mathrm{e}-1$ & 0.9 & $3.8 \mathrm{e}-1$ & 0.7 & $1.5 \mathrm{e}-1$ & 1.0 & $1.3 \mathrm{e}-1$ & 1.0 \\
\hline & 0.08 & $1.0 \mathrm{e}-1$ & 1.0 & $1.8 \mathrm{e}-1$ & 1.1 & $5.8 \mathrm{e}-2$ & 1.3 & $5.4 \mathrm{e}-2$ & 1.3 \\
\hline & 0.04 & $3.2 \mathrm{e}-2$ & 1.6 & $7.2 \mathrm{e}-2$ & 1.3 & $1.6 \mathrm{e}-2$ & 1.9 & $1.5 \mathrm{e}-2$ & 1.8 \\
\hline \multirow{5}{*}{$\beta=0:$} & $\triangle x$ & $e_{v}$ & rate & $e_{q}$ & rate & $e_{U}$ & rate & $e_{\eta}$ & rate \\
\hline & 0.34 & $3.7 \mathrm{e}-1$ & - & $6.1 \mathrm{e}-1$ & - & $3.1 \mathrm{e}-1$ & - & $2.7 \mathrm{e}-1$ & - \\
\hline & 0.17 & $2.0 \mathrm{e}-1$ & 0.9 & $3.7 e-1$ & 0.7 & $1.5 \mathrm{e}-1$ & 1.0 & $1.4 \mathrm{e}-1$ & 0.9 \\
\hline & 0.08 & $1.0 \mathrm{e}-1$ & 1.0 & $1.8 \mathrm{e}-1$ & 1.0 & $6.0 \mathrm{e}-2$ & 1.3 & $5.5 \mathrm{e}-2$ & 1.3 \\
\hline & 0.04 & $3.6 \mathrm{e}-2$ & 1.5 & $6.8 \mathrm{e}-2$ & 1.4 & $1.7 \mathrm{e}-2$ & 1.8 & $1.5 \mathrm{e}-2$ & 1.9 \\
\hline
\end{tabular}

Table 3: Convergence in space. Top: $\beta=1$. Bottom: $\beta=0$.

where $\nabla_{\gamma}(\cdot)$ denotes the surface gradient. Practically, the surface gradient can be computed as $[23,10]$

$$
\nabla_{\gamma}(\boldsymbol{\eta})=\nabla \boldsymbol{\eta}(\boldsymbol{I}-\boldsymbol{n} \otimes \boldsymbol{n})
$$

where the symbol $\otimes$ denotes the tensor product and $\boldsymbol{I}$ is the identity operator. The coefficients $E$ and $\sigma$ are the Young's modulus and the Poisson's ratio for the membrane, respectively. This model was obtained from a three-dimensional elasticity model after assuming a thin- 
wall structure, a homogeneous distribution of radial stresses and negligible bending terms. Comparisons between this model, other reduced order models and a full 3D model for fluid - structure interaction problems was investigated in [23]. The advantage of this model compared to other reduced models is that the displacement is considered non zero in all the three spatial directions.

As in Example 1, the flow is driven by the time-dependent pressure data

$$
p_{\text {in }}(t)=\left\{\begin{array}{ll}
\frac{p_{\max }}{2}\left(1-\cos \left(\frac{2 \pi t}{t_{\text {max }}}\right)\right) & \text { it } t \leq t_{\text {max }}, \\
0 & \text { it } t>t_{\text {max }},
\end{array} \quad p_{\text {out }}(t)=0, \forall t \in(0, T),\right.
$$

where $p_{\max }=2 \cdot 10^{4}$ dyne $/ \mathrm{cm}^{2}$ and $t_{\max }=5 \mathrm{~ms}$. The problem was solved with a time step $\Delta t=10^{-4}$ over the time interval $[0,14] \mathrm{ms}$. The reference domain $\Omega=\Omega^{f} \cup \Omega^{p}$ is a cylinder of length $L$ and radius $R$. Reference domains $\Omega^{f}$ and $\Omega^{p}$ are shown in Figure 7 . We

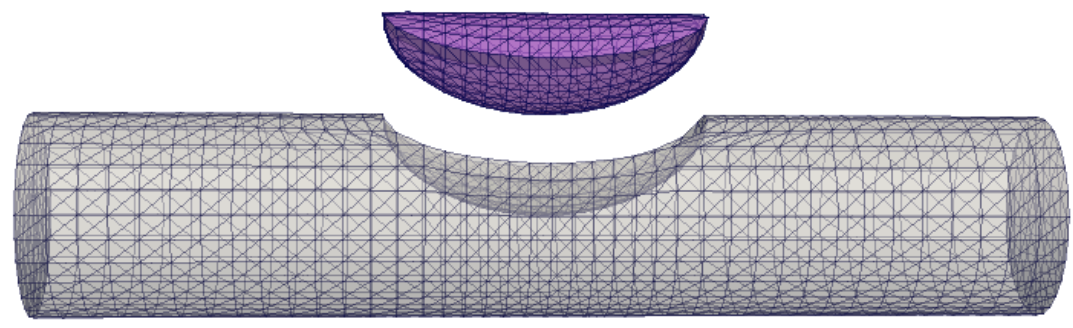

Figure 7: Top: Reference poroelastic domain $\Omega^{p}$. Bottom: Reference fluid domain $\Omega^{f}$.

used P1 bubble - P1 elements to approximate the fluid velocity and pressure, P1 elements to approximate the thin structure and poroelastic material displacement, and P2 and P1 elements to approximate the Darcy velocity and pressure. The fluid mesh contains 3541 nodes and 15852 cells, and the poroelastic medium mesh contains 812 nodes and 2839 cells. The value of $\beta$ in this example is $\beta=1$, and the values of the combination parameters are $\alpha_{s}=10^{-3}, \alpha_{p}=10^{-3}$ and $\alpha_{f}=10^{4}$.

In this example, we focus on investigating the effects of the material properties of the poroelastic region on the fluid flow. We consider three different cases, based on the values of the storativity (the inverse of the Biot's modulus) and permeability coefficients:

$$
\begin{aligned}
& \text { CASE 1: } \quad \frac{1}{M}=10^{-3} \mathrm{~cm}^{2} / \text { dyne }, \quad \boldsymbol{\kappa}=10^{-3} \boldsymbol{I} \mathrm{cm}^{3} \mathrm{~s} / \mathrm{g}, \\
& \text { CASE 2: } \quad \frac{1}{M}=10^{-6} \mathrm{~cm}^{2} / \text { dyne, } \quad \boldsymbol{\kappa}=10^{-3} \boldsymbol{I} \mathrm{cm}^{3} \mathrm{~s} / \mathrm{g} \text {, } \\
& \text { CASE 3: } \quad \frac{1}{M}=10^{-3} \mathrm{~cm}^{2} / \text { dyne }, \quad \boldsymbol{\kappa}=10^{-6} \boldsymbol{I} \mathrm{cm}^{3} \mathrm{~s} / \mathrm{g} .
\end{aligned}
$$

The values of the other parameters used in this example are given in Table 4 . The slip rate $\gamma^{f p}$ is calculated according to formula [19]

$$
\gamma^{f p}=\frac{\alpha \mu_{f} \sqrt{3}}{\sqrt{\mu_{f} \operatorname{trace}(\boldsymbol{\kappa})}} .
$$




\begin{tabular}{|c|c|c|c|}
\hline Parameters & Values & Parameters & Values \\
\hline $\begin{array}{l}\text { Geometry: } \\
\text { Radius } R(\mathrm{~cm})\end{array}$ & 0.5 & Length $L(\mathrm{~cm})$ & 5 \\
\hline $\begin{array}{l}\text { Fluid: } \\
\text { Fluid density } \rho_{f}\left(\mathrm{~g} / \mathrm{cm}^{3}\right)\end{array}$ & 1 & Fluid viscosity $\mu_{f}(\mathrm{~g} / \mathrm{cm} \mathrm{s})$ & 0.035 \\
\hline $\begin{array}{l}\text { Thin structure (membrane): } \\
\text { Membrane thickness } h(\mathrm{~cm}) \\
\text { Young's modulus } E\left(\text { dyne } / \mathrm{cm}^{2}\right)\end{array}$ & $\begin{array}{l}0.1 \\
10^{6}\end{array}$ & $\begin{array}{l}\text { Membrane density } \rho_{m}\left(\mathrm{~g} / \mathrm{cm}^{3}\right) \\
\text { Poisson's ratio } \sigma\end{array}$ & $\begin{array}{l}1.1 \\
0.5\end{array}$ \\
\hline $\begin{array}{l}\text { Poroelasticity: } \\
\text { Porosity } \phi\end{array}$ & 0.9 & Biot-Willis constant $\alpha$ & 1 \\
\hline $\begin{array}{l}\text { Fluid density } \rho_{q}\left(\mathrm{~g} / \mathrm{cm}^{3}\right) \\
\text { Lamé coeff. } \mu_{s}\left(\text { dyne } / \mathrm{cm}^{2}\right)\end{array}$ & $\begin{array}{l}1.027 \\
2.227 \times 10^{5}\end{array}$ & $\begin{array}{l}\text { Structure density } \rho_{s}\left(\mathrm{~g} / \mathrm{cm}^{3}\right) \\
\text { Lamé coeff. } \lambda_{s}\left(\text { dyne } / \mathrm{cm}^{2}\right)\end{array}$ & $\begin{array}{l}1.3 \\
2.398 \times 10^{9}\end{array}$ \\
\hline
\end{tabular}

Table 4: Geometry, fluid, structure, and poroelasticity parameters used in Example 2.

In the existing literature, due to the complexity of the problem, the flow past a thrombotic region in medium to large arteries is often modeled by neglecting the dynamics of the thrombus. In order to show the importance of including the model for the poroelastic region, we compare our results to the ones obtained by solving the FSI problem only. Namely, we solve the flow problem where the reference domain is the same as domain $\Omega^{f}$ shown in Figure 7 , but the whole lateral boundary is modeled as a linearly elastic membrane described by equation (5.4). This problem is solved using the kinematically coupled $\beta$-scheme. Figure 8 shows the comparison of the fluid pressure and the velocity vectors obtained in the high storativity regime (Case 1), low storativity regime (Case 2), and using the FSI model only. We observe that changing the storativity coefficient, which is related to the compressibility of the poroelastic system, causes a significant difference in the fluid flow.

The fluid pressure dissipates more in the high storativity regime, while the Darcy pressure is larger in the low storativity case. In both cases the pressure dissipation is higher than in the flow obtained by modeling FSI without taking into account the dynamics of the poroelastic region. We also observe a significant difference between the velocity vectors obtained in Cases 1 and 2, and in the case of modeling FSI only.

In the high permeability regime (Case 1) and the low permeability regime (Case 3 ), for the same value of the storativity coefficient, we do not observe major changes in the fluid pressure in the cylinder. However, the high permeability regime leads to the increase of the fluid velocity close to the fluid - poroelastic material interface, as well as the increase of the Darcy velocity. The fluid pressure, Darcy pressure, and the velocity vectors at $t=11 \mathrm{~ms}$ are shown in the left panel in Figure 9. The Darcy velocity in the two cases at $t=11 \mathrm{~ms}$ is shown on the right panel in Figure 9.

Finally, we compare the displacement of the poroelastic material in three different test cases. Figure 10 shows the magnitude of the displacement and the displacement vectors at $t=8 \mathrm{~ms}$ and $t=11 \mathrm{~ms}$. The displacement is the largest in the low storativity regime (Case 2). Furthermore, while the displacement at $t=11 \mathrm{~ms}$ in Cases 1 and 3 is mostly in the longitudinal direction, due to the higher Darcy pressure the displacement in Case 2 still features significant radial motion. 


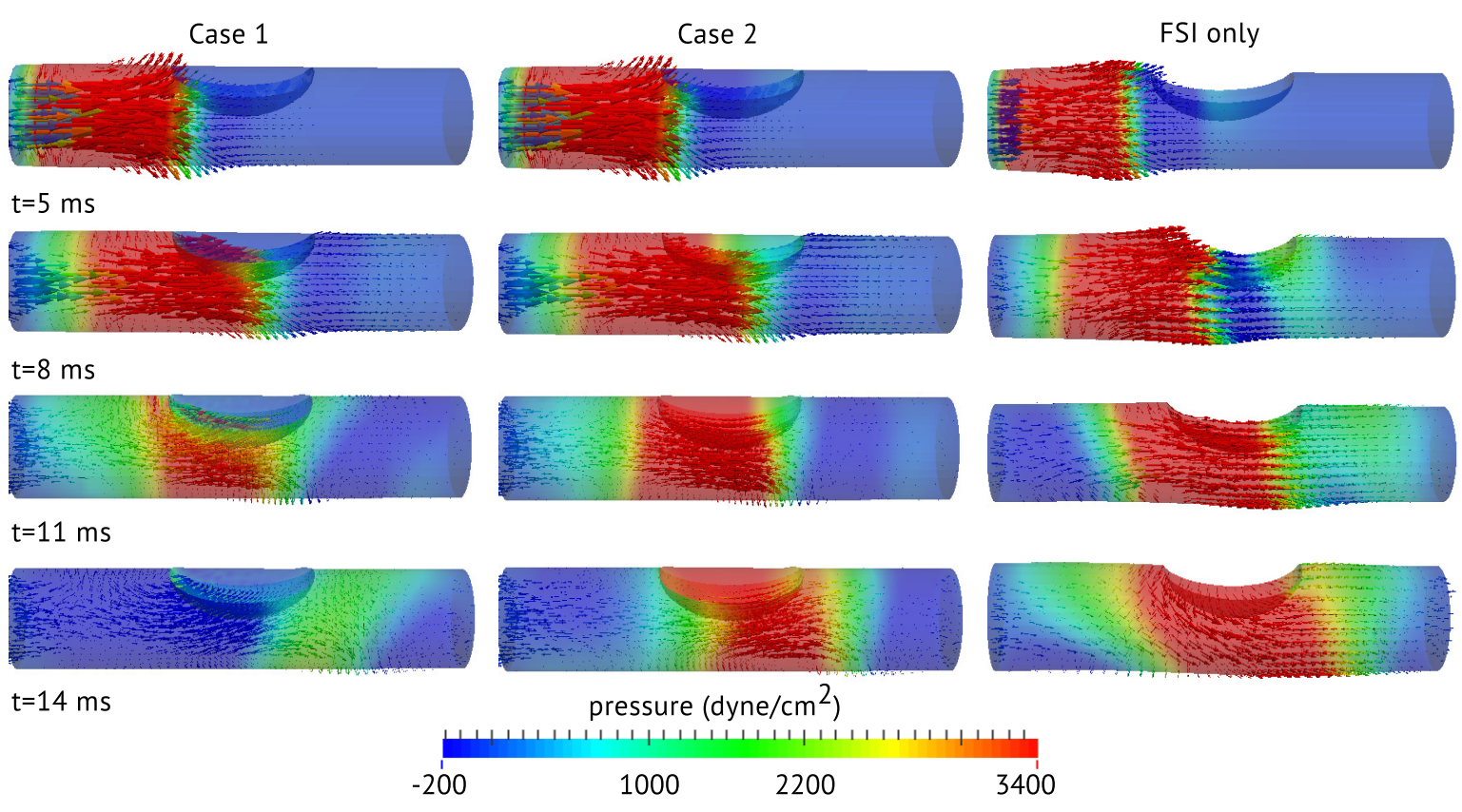

Figure 8: Propagation of the fluid pressure wave, Darcy pressure, and the velocity vectors at four different times. First column corresponds to the high storativity regime (Case 1), second column to the low storativity regime (Case 2), and the third column to the model of the fluid-structure interaction only. We observe significant differences in the flow in all three cases.

\section{Conclusions}

In this work we present a novel model and a new loosely coupled numerical scheme for the interaction between an incompressible, viscous fluid, thin elastic structure and a poroelastic material. The poroelastic material is modeled using the dynamic Biot equations, in which case the system can not be reduced to the primal, pressure - displacement formulation. This choice of the model leads to energy estimates of the coupled problem which, to best of our knowledge, have not been reported before.

We analyze the stability of the numerical method and show a conditional stability under an assumption on the combination parameters $\alpha_{f}, \alpha_{s}$ and $\alpha_{p}$. The scheme is implemented in $2 \mathrm{D}$ and $3 \mathrm{D}$ using two different models for the thin structure displacement. Our numerical tests indicate the first order convergence in time when $\beta=1$ and the first order convergence in space. Similar to the kinematically coupled $\beta$-scheme for FSI problems, parameter $\beta$ is shown to increase the accuracy in time without affecting the stability of the scheme.

Lastly, we investigate the effects of the material properties of the poroelastic region on the fluid flow. We show that different values of the storativity coefficient (the inverse of the Biot's modulus) and permeability lead to significant differences in the fluid flow. Increased fluid pressure is observed in the low storativity regime, while increased fluid velocity in the vicinity of the fluid-poroelastic material interface is observed in the high permeability/high storativity regime. Comparison of our results with the ones obtained by neglecting the dynamics of the poroelastic material show that different values of the velocity and pressure are obtained by 


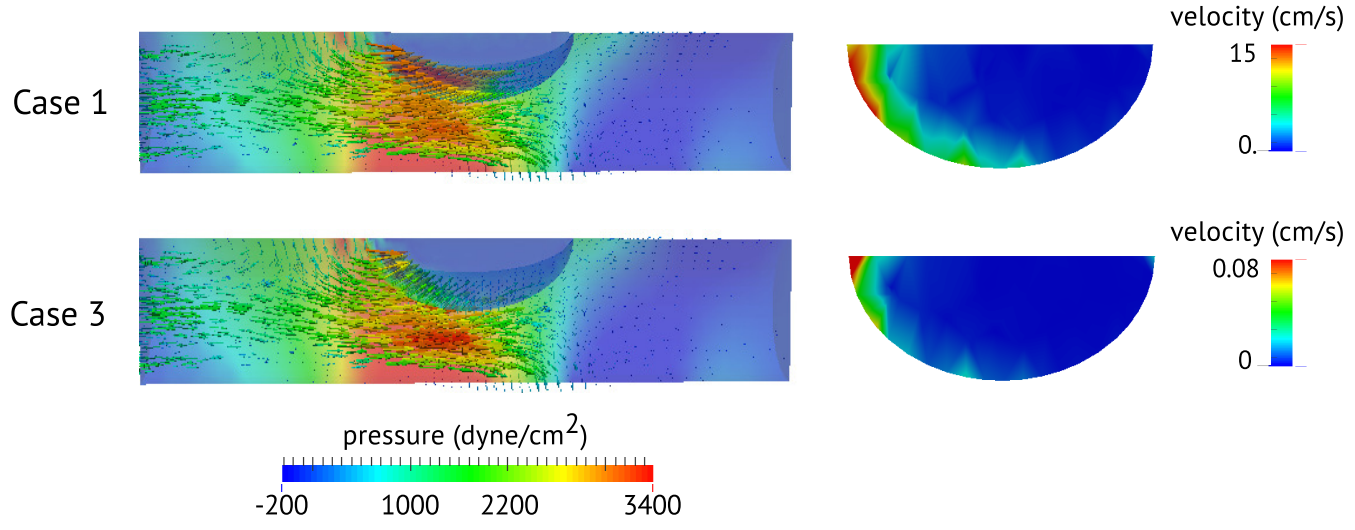

Figure 9: Left: Fluid pressure, Darcy pressure and velocity vectors at $t=11 \mathrm{~ms}$ in the high permeability regime (top) and the low permeability regime (bottom), obtained with the same value of the storativity coefficient. Right: Darcy velocity at $t=11 \mathrm{~ms}$ in the high permeability regime (top) and the low permeability regime (bottom). We notice the significant decrease of the velocity as we decrease the permeability.

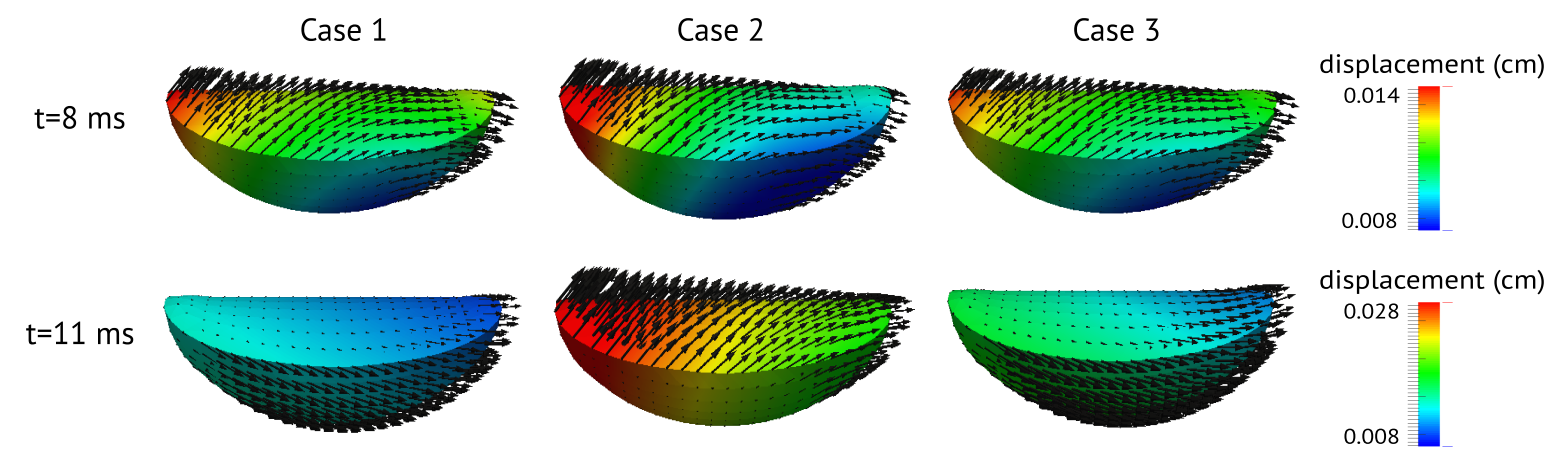

Figure 10: The magnitude of the displacement (colorbar) of the poroelastic material at $t=8 \mathrm{~ms}$ (top) and $t=11 \mathrm{~ms}$ (bottom). The displacement is superimposed with the displacement vectors. We notice that the displacement is the largest in the low storativity regime (Case 2).

modeling the fluid-structure interaction only. Hence, the computation of shear rates, which are often of interest in biomedical applications, significantly differs if the dynamics of the poroelastic material is not taken into account.

One of the drawbacks of the method proposed here is that the stability and accuracy of the scheme rely on the appropriate quantification of the combination parameters $\alpha_{f}, \alpha_{s}$ and $\alpha_{p}$. Furthermore, the convergence to a strongly coupled scheme has only been numerically investigated in the case of rigid walls since no monolithic or strongly coupled algorithms have been proposed for the full problem. To properly quantify the effects of the combination parameters on the accuracy of the solution, we will consider derivation of the a priori error estimates, development of the preconditioners for the monolithic system and comparison of our results to the ones obtained using a monolithic approach in our future work. 


\section{Acknowledgements}

The author would like to thank the anonymous reviewers for their comments that help improve the manuscript. Research of Bukač was partially supported by NSF via grant DMS1318763 and by NIH via grant U01 HL116330-01.

\section{References}

[1] R. Adolph, D. Vorp, D. Steed, M. Webster, M. Kameneva, and S. Watkins. Cellular content and permeability of intraluminal thrombus in abdominal aortic aneurysm. Journal of vascular surgery, 25(5):916-926, 1997.

[2] J. Allard and N. Atalla. Propagation of Sound in Porous Media: Modelling Sound Absorbing Materials 2e. John Wiley \& Sons, 2009.

[3] N. Atalla, M. Hamdi, and R. Panneton. Enhanced weak integral formulation for the mixed $\left(\mathrm{u}_{-}, \mathrm{p}_{-}\right)$poroelastic equations. The Journal of the Acoustical Society of America, 109(6):3065-3068, 2001.

[4] N. Atalla, R. Panneton, and P. Debergue. A mixed displacement-pressure formulation for poroelastic materials. The Journal of the Acoustical Society of America, 104:1444-1452, 1998.

[5] S. Badia, F. Nobile, and C. Vergara. Fluid-structure partitioned procedures based on Robin transmission conditions. Journal of Computational Physics, 227:7027-7051, 2008.

[6] S. Badia, F. Nobile, and C. Vergara. Robin-Robin preconditioned Krylov methods for fluid-structure interaction problems. Computer Methods in Applied Mechanics and Engineering, 198(33-36):2768-2784, 2009.

[7] S. Badia, A. Quaini, and A. Quarteroni. Splitting methods based on algebraic factorization for fluid-structure interaction. SIAM Journal on Scientific Computing, 30(4):1778$1805,2008$.

[8] S. Badia, A. Quaini, and A. Quarteroni. Coupling Biot and Navier-Stokes equations for modelling fluid-poroelastic media interaction. Journal of Computational Physics, 228(21):7986-8014, 2009.

[9] A.T. Barker and X.C. Cai. Scalable parallel methods for monolithic coupling in fluidstructure interaction with application to blood flow modeling. Journal of Computational Physics, 229(3):642-659, 2010.

[10] A. Bonito, R. Nochetto, and M. Pauletti. Dynamics of biomembranes: effect of the bulk fluid. Mathematical modelling of natural phenomena, 6(05):25-43, 2011.

[11] M. Bukač, S. Čanić, R. Glowinski, J. Tambača, and A. Quaini. Fluid-structure interaction in blood flow capturing non-zero longitudinal structure displacement. Journal of Computational Physics, 235:515-541, 2013. 
[12] M. Bukač, S. Čanić, and B. Muha. A partitioned scheme for fluid-composite structure interaction problems. Journal of Computational Physics, 281:493-517, 2015.

[13] M. Bukač, I. Yotov, and P. Zunino. An operator splitting approach for the interaction between a fluid and a multilayered poroelastic structure. Numerical Methods for Partial Differential Equations, 31(4):1054-1100, 2015.

[14] E. Burman and M. A. Fernández. Stabilization of explicit coupling in fluid-structure interaction involving fluid incompressibility. Computer Methods in Applied Mechanics and Engineering, 198:766-784, 2009.

[15] E. Burman and Fernández M.A. An unfitted Nitsche method for incompressible fluidstructure interaction using overlapping meshes. Computer Methods in Applied Mechanics and Engineering, 279:497 - 514, 2014.

[16] S. Canic, B. Muha, and M. Bukač. Stability of the kinematically coupled $\beta$-scheme for fluid-structure interaction problems in hemodynamics. International Journal of Numerical Analysis and Modeling, 12(1):54-80, 2015.

[17] Y. Cao, M. Gunzburger, X. He, and X. Wang. Robin-Robin domain decomposition methods for the steady-state Stokes-Darcy system with the Beavers-Joseph interface condition. Numerische Mathematik, 117(4):601-629, 2011.

[18] Y. Cao, M. Gunzburger, X. He, and X. Wang. Parallel, non-iterative, multi-physics domain decomposition methods for time-dependent Stokes-Darcy systems. Mathematics of Computation, 83(288):1617-1644, 2014.

[19] Y. Cao, M. Gunzburger, X. Hu, F. Hua, X. Wang, and W. Zhao. Finite element approximations for Stokes-Darcy flow with Beavers-Joseph interface conditions. SIAM Journal on Numerical Analysis, 47(6):4239-4256, 2010.

[20] P. Causin, J.F. Gerbeau, and F. Nobile. Added-mass effect in the design of partitioned algorithms for fluid-structure problems. Computer Methods in Applied Mechanics and Engineering, 194(42-44):4506-4527, 2005.

[21] J. Chen. Time domain fundamental solution to Biot's complete equations of dynamic poroelasticity. Part II: Three-dimensional solution. International Journal of Solids and Structures, 31(2):169-202, 1994.

[22] W. Chen, M. Gunzburger, F. Hua, and X. Wang. A parallel Robin-Robin domain decomposition method for the Stokes-Darcy system. SIAM Journal on Numerical Analysis, 49(3):1064-1084, 2011.

[23] C. Colciago, S. Deparis, and A. Quarteroni. Comparisons between reduced order models and full 3D models for fluid-structure interaction problems in haemodynamics. Journal of Computational and Applied Mathematics, 265:120-138, 2014.

[24] P. Debergue, R. Panneton, and N. Atalla. Boundary conditions for the weak formulation of the mixed $(\mathrm{u}, \mathrm{p})$ poroelasticity problem. The Journal of the Acoustical Society of America, 106(5):2383-2390, 1999. 
[25] J. Donea. Arbitrary Lagrangian-Eulerian finite element methods, in: Computational methods for transient analysis. North-Holland, Amsterdam, 1983.

[26] W. Ehlers and B. Markert. A linear viscoelastic biphasic model for soft tissues based on the theory of porous media. Journal of biomechanical engineering, 123(5):418-424, 2001.

[27] M. Fernández and M. Landajuela. A fully decoupled scheme for the interaction of a thin-walled structure with an incompressible fluid. Comptes Rendus Mathématique, 351(3):161-164, 2013.

[28] M.A. Fernández. Incremental displacement-correction schemes for incompressible fluidstructure interaction: stability and convergence analysis. Numerische Mathematik, $123: 210-65,2012$.

[29] M.A. Fernández and J. Mullaert. Displacement-velocity correction schemes for incompressible fluid-structure interaction. Comptes Rendus Mathématique, 349(17-18):1011$1015,2011$.

[30] A. Figueroa, S. Baek, C. Taylor, and J. Humphrey. A computational framework for fluid-solid-growth modeling in cardiovascular simulations. Computer Methods in Applied Mechanics and Engineering, 198(45):3583-3602, 2009.

[31] A. Figueroa, I.E. Vignon-Clementel, K. Jansen, T. Hughes, and C. Taylor. A coupled momentum method for modeling blood flow in three-dimensional deformable arteries. Computer Methods in Applied Mechanics and Engineering, 195(41-43):5685-5706, 2006.

[32] M. Folkesson, M. Kazi, C. Zhu, A. Silveira, A.-L. Hemdahl, A. Hamsten, U. Hedin, J. Swedenborg, P. Eriksson, et al. Presence of NGAL/MMP-9 complexes in human abdominal aortic aneurysms. Thrombosis and Haemostasis, 98(2):427-433, 2007.

[33] L. Formaggia, J.F. Gerbeau, F. Nobile, and A. Quarteroni. On the coupling of 3D and 1D Navier-Stokes equations for flow problems in compliant vessels. Computer Methods in Applied Mechanics and Engineering, 191(6-7):561-582, 2001.

[34] X. Gai. A coupled geomechanics and reservoir flow model on parallel computers. PhD thesis, University of Texas at Austin, 2004.

[35] B. Ganis, V. Girault, M. Mear, G. Singh, and M. Wheeler. Modeling fractures in a poro-elastic medium. Oil \& Gas Science and Technology-Revue dIFP Energies nouvelles, 2013.

[36] C. Gasser, G. Martufi, M. Auer, M. Folkesson, and J. Swedenborg. Micromechanical characterization of intra-luminal thrombus tissue from abdominal aortic aneurysms. Annals of biomedical engineering, 38(2):371-379, 2010.

[37] V. Girault, M. Wheeler, B. Ganis, and M. Mear. A lubrication fracture model in a poro-elastic medium. Mathematical Models and Methods in Applied Sciences, 1:1-59, 2014. 
[38] G. Guidoboni, R. Glowinski, N. Cavallini, and S. Čanić. Stable loosely-coupled-type algorithm for fluid-structure interaction in blood flow. Journal of Computational Physics, 228(18):6916-6937, 2009.

[39] P. Hansbo. Nitsche's method for interface problems in computational mechanics. GAMM-Mitteilungen, 28(2):183-206, 2005.

[40] F. Hecht. New development in FreeFem++. Journal of Numerical Mathematics, 20(34):251-265, 2012.

[41] T.J.R. Hughes, W.K. Liu, and T.K. Zimmermann. Lagrangian-Eulerian finite element formulation for incompressible viscous flows. Computer Methods in Applied Mechanics and Engineering, 29(3):329-349, 1981.

[42] A. Hundertmark-Zaušková, M. Lukácová-Medvidová, and G. Rusnáková. Fluidstructure interaction for shear-dependent non-Newtonian fluids. Topics in mathematical modeling and analysis, 7:109-158, 2012.

[43] S. Jonckheere, D. Vandepitte, and W. Desmet. Modelling of sound damping multi-layers using a hybrid finite element-wave based method. In Proceedings of the 25th Conference on Noise and Vibration Engineering (ISMA2012), volume 25, pages 4129-4142, 2012.

[44] I. Karšaj and J. Humphrey. A mathematical model of evolving mechanical properties of intraluminal thrombus. Biorheology, 46(6):509, 2009.

[45] N. Koshiba, J. Ando, X. Chen, and T. Hisada. Multiphysics simulation of blood flow and LDL transport in a porohyperelastic arterial wall model. Journal of biomechanical engineering, 129(3):374-385, 2007.

[46] J. Lee, Y. Kang, and Y. Kim. Unified multiphase modeling for evolving, acoustically coupled systems consisting of acoustic, elastic, poroelastic media and septa. Journal of Sound and Vibration, 331(25):5518-5536, 2012.

[47] M. Lukáčová-Medvid'ová, G. Rusnáková, and A. Hundertmark-Zaušková. Kinematic splitting algorithm for fluid-structure interaction in hemodynamics. Computer Methods in Applied Mechanis and Engineering, 265:83-106, 2013.

[48] P. Matuszyk and L. Demkowicz. Solution of coupled poroelastic/acoustic/elastic wave propagation problems using automatic hp-adaptivity. Computer Methods in Applied Mechanics and Engineering, 281:54 - 80, 2014.

[49] A. Mikelić and M. Wheeler. Convergence of iterative coupling for coupled flow and geomechanics. Computational Geosciences, 17(3):455-461, 2013.

[50] L.A. Miller and C.S. Peskin. A computational fluid dynamics of 'clap and fling' in the smallest insects. Journal of Experimental Biology, 208(2):195-212, 2005.

[51] B. Muha and S. Canić. Existence of a Weak Solution to a Nonlinear Fluid-Structure Interaction Problem Modeling the Flow of an Incompressible, Viscous Fluid in a Cylinder with Deformable Walls. Archive for Rational Mechanics and Analysis, 207(3):919-968, 2013. 
[52] B. Muha and S. Čanić. Existence of a weak solution to a fluid-multi-layered-structure interaction problem. Journal of Differential Equations, 256(2):658-706, 2014.

[53] M. A. Murad, J. N. Guerreiro, and A. F.D. Loula. Micromechanical computational modeling of secondary consolidation and hereditary creep in soils. Computer Methods in Applied Mechanics and Engineering, 190(15):1985-2016, 2001.

[54] F. Nobile. Numerical approximation of fluid-structure interaction problems with application to haemodynamics. PhD thesis, EPFL, Switzerland, 2001.

[55] F. Nobile and C. Vergara. An effective fluid-structure interaction formulation for vascular dynamics by generalized Robin conditions. SIAM Journal on Scientific Computing, 30:731-763, 2008.

[56] P. Phillips and M. Wheeler. A coupling of mixed and discontinuous Galerkin finiteelement methods for poroelasticity. Computational Geosciences, 12(4):417-435, 2008.

[57] S. Polzer, C. Gasser, B. Markert, J. Bursa, and P. Skacel. Impact of poroelasticity of intraluminal thrombus on wall stress of abdominal aortic aneurysms. BioMedical Engineering online, 11:62, 2012.

[58] A. Quaini and A Quarteroni. A semi-implicit approach for fluid-structure interaction based on an algebraic fractional step method. Mathematical Models and Methods in Applied Sciences, 17(6):957-985, 2007.

[59] S. Rigobert, F. Sgard, and N. Atalla. A two-field hybrid formulation for multilayers involving poroelastic, acoustic, and elastic materials. The Journal of the Acoustical Society of America, 115(6):2786-2797, 2004.

[60] R. Showalter. Poroelastic filtration coupled to Stokes flow. Lecture Notes in Pure and Applied Mathematics, 242:229-241, 2010.

[61] R. Showalter and B. Momken. Single-phase flow in composite poroelastic media. Mathematical methods in the applied sciences, 25(2):115-139, 2002.

[62] R. Showalter and U. Stefanelli. Diffusion in poro-plastic media. Mathematical methods in the applied sciences, 27(18):2131-2151, 2004.

[63] B. Tully and Y. Ventikos. Coupling poroelasticity and CFD for cerebrospinal fluid hydrodynamics. Biomedical Engineering, IEEE Transactions on, 56(6):1644-1651, 2009.

[64] M. Wheeler, G. Xue, and I. Yotov. Coupling multipoint flux mixed finite element methods with continuous Galerkin methods for poroelasticity. Computational Geosciences, 18(1):57-75, 2014.

[65] Z. Xu, J. Lioi, J. Mu, M. Kamocka, X. Liu, D. Chen, E. Rosen, and M. Alber. A multiscale model of venous thrombus formation with surface-mediated control of blood coagulation cascade. Biophysical journal, 98(9):1723-1732, 2010.

[66] D. Yang and Z. Zhang. Poroelastic wave equation including the Biot/squirt mechanism and the solid/fluid coupling anisotropy. Wave Motion, 35(3):223-245, 2002. 
[67] T. Zielinski. Finite-Element Modelling of Fully-Coupled Active Systems Involving Poroelasticity, Piezoelectricity, Elasticity, and Acoustics. In Proceedings of the 19th International Conference on Computer Methods in Mechanics CMM2011, Warsaw, Poland, 2011.

[68] O. Zienkiewicz, C. Chang, and P. Bettess. Drained, undrained, consolidating and dynamic behaviour assumptions in soils. Geotechnique, 30(4):385-395, 1980. 